\title{
Investigating the Effect of Sensory Concurrency on Learning Haptic Spatiotemporal Signals
}

\author{
IAIN CARSON, UTA HINRICHS, and LORAINE CLARKE, University of St Andrews \\ AARON QUIGLEY, UNSW Sydney
}

A new generation of multimodal interfaces and interactions is emerging. Drawing on the principles of Sensory Substitution and Augmentation Devices (SSADs), these new interfaces offer the potential for rich, immersive human-computer interactions, but are difficult to design well, and take time to master, creating significant barriers towards wider adoption. Following a review of the literature surrounding existing SSADs, their metrics for success and their growing influence on interface design in Human Computer Interaction, we present a medium term (4-day) study comparing the effectiveness of various combinations of visual and haptic feedback (sensory concurrencies) in preparing users to perform a virtual maze navigation task using haptic feedback alone. Participants navigated 12 mazes in each of 3 separate sessions under a specific combination of visual and haptic feedback, before performing the same task using the haptic feedback alone. Visual sensory deprivation was shown to be inferior to visual \& haptic concurrency in enabling haptic signal comprehension, while a new hybridized condition combining reduced visual feedback with the haptic signal was shown to be superior. Potential explanations for the effectiveness of the hybrid mechanism are explored, and the scope and implications of its generalization to new sensory interfaces is presented.

CCS Concepts: • Human-centered computing $\rightarrow$ HCI theory, concepts and models; User studies; Empirical studies in HCI; Interaction design theory, concepts and paradigms; Haptic devices.

Additional Key Words and Phrases: sensory, learning, interface, haptics, augmentation, vibrotactile

\section{ACM Reference Format:}

Iain Carson, Uta Hinrichs, Loraine Clarke, and Aaron Quigley. 2021. Investigating the Effect of Sensory Concurrency on Learning Haptic Spatiotemporal Signals. Proc. ACM Interact. Mob. Wearable Ubiquitous Technol. 0, 0, Article 0 ( 2021), 32 pages. https://doi.org/10. $1145 / 1122445.1122456$

Authors' addresses: Iain Carson, ic48@st-andrews.ac.uk; Uta Hinrichs, uh3@st-andrews.ac.uk; Loraine Clarke, lec24@st-andrews.ac.uk, University of St Andrews, KY16 9SX; Aaron Quigley, a.quigley@unsw.edu.au, UNSW Sydney, New South Wales, AU.

Permission to make digital or hard copies of all or part of this work for personal or classroom use is granted without fee provided that copies are not made or distributed for profit or commercial advantage and that copies bear this notice and the full citation on the first page. Copyrights for components of this work owned by others than ACM must be honored. Abstracting with credit is permitted. To copy otherwise, or republish, to post on servers or to redistribute to lists, requires prior specific permission and/or a fee. Request permissions from permissions@acm.org.

๑ 2021 Association for Computing Machinery.

Manuscript submitted to ACM

Manuscript submitted to ACM 


\section{INTRODUCTION}

Recent advances in human computer interaction have seen the integration of multiple modalities into a single interface, simultaneously stimulating multiple user senses to provide task-relevant information. In particular, advanced haptic interfaces have emerged that can allow for more immersive experiences or allow for communication of complex information. In this paper we focus in particular on Vibrotactile Sensory Substitution and Augmentation Devices (SSADs), i.e. devices that have been designed to augment or substitute sensory experiences using dynamic, continuous, spatio-temporal signals in response to the user's interactions in a (physical or virtual) environment. Previous work in this area has introduced such interfaces to facilitate demanding tasks in the context of Sensory Substitution and Augmentation (such as sensory impairment aids like the Neosensory Buzz [39] which allows feeling sounds on the arm, or related efforts by Zhao [51] which targets language directly) or driving (The Lateral Line [25] augments driver awareness directly, and Feeling Uncertain [24] arms users with a dynamic awareness of semi-autonomous car sensor uncertainty). Some attempts have been made to produce toolkits for general creation of sensory signals with a variety of input mechanisms, such as TESSA [46] and the TongueDuino [11]. Several devices have been created specifically to further our understanding of how such dynamic vibrotactile signals may be integrated alongside our natural senses, such as The Enactive Torch [14] and Feelspace Belt [38] which have led to several involved studies and are addressed later in detail.

Leveraging the haptic sense to represent continuous and fast-changing information (e.g., the proximity to a target or speed of movement), is a challenge. As human beings we are experts in the visual interpretation of such information, but efforts to accurately interpret haptic feedback have proven a difficult and time-consuming process which requires both intensive training and/or long-term exposure [1,33]. Due to the abstract nature of the information in the signal, there is often a steep learning curve associated with these devices, long-recognized as a barrier to uptake [4, 15, 23, 43]. Common consumer interfaces (e.g., games controllers, mobile phones, or smart watches) that make use of haptic feedback typically avoid this problem by (1) limiting the complexity of the signal and the information to be represented (e.g., discrete information about system status represented as a small number of distinct haptic symbols or tactons [5]), or by (2) using the haptic modality as a subordinate channel to improve immersion rather than communicating crucial information (such as the Nintendo Switch's HD controller rumble [26]).

Development of "intuitive" mappings between information and haptic feedback has been shown to facilitate and speed up learning processes of haptic encodings [8, 16]. However, a broad review of existing training strategies for sensory augmentation devices by Bertram and Stafford has shown that we still lack systematic approaches to teaching information encodings in haptic signals. Specifically, we lack understanding of how feedback and learning conditions contribute to the development of competence and people's ability to effectively interpret encodings in-situ [4].

To ground the development of our hypothesis, our area of focus is on the role that concurrent sensory information plays in enabling and encouraging such learning. To illustrate, full "sensory concurrency", where duplicate information is received across more than one sense, provides a feedback channel which may be critical for the learning of dynamic haptic signals commonly used by SSADs $[4,20]$. However, "sensory deprivation", where task-critical senses such as vision are inhibited, forces user attention towards the new signal and creates incentives which also promote learning [32]. These opposing paradigms have both been shown in isolation to contribute to successful learning of SSAD signals, however, we believe that understanding and optimizing the role of both sensory concurrency and sensory deprivation during SSAD learning is key to improving the training process. As a result, we hypothesize that both rich feedback and 
an incentive to learn can be combined through careful consideration of both paradigms, and that SSAD signal learning may be accelerated when training under such conditions.

In this research we start addressing these questions by actively investigating how the visual sense can be leveraged to facilitate the decoding of vibrotactile information as part of spatio-temporal tasks. In particular, we report the results of an experiment where we compare combinations of visual and haptic signals conveying task-relevant information to participants. Our findings demonstrate how the various combinations of multimodal concurrency affects the interpretation of a vibrotactile spatio-temporal information in a maze navigation task. This interaction between the visual and haptic sense points towards mechanisms that can be employed to improve decoding and interpretation of vibrotactile information, ultimately reducing the overheads for widespread adoption of sophisticated haptic interfaces.

We detail the systematic comparison of the performance and sentiment of users executing a dynamic, virtual maze navigation task using only a novel haptic feedback signal. The users first execute the task unguided and unsupervised over a medium-term study, under one of several visual and haptic feedback combinations. Each combination reflects a specific hypothesized mechanism for learning through sensory concurrency and deprivation. Users in all conditions were unaware that the final task required navigation using solely haptic feedback, maximizing their efforts to complete the task, rather than to learn the haptic signal. Therefore, the results provide an indication of learning effectiveness of each combination of modalities independent of deliberated attention.

The presented experimental design gives insights into how comprehension of new dynamic spatio-temporal vibrotactile signals is acquired. We develop these insights into principles that promote effective procedural learning during normal, productive task execution. Our principles offer an alternative to dedicated training (such as feedback on training task completion, or dynamic user-controlled feedback systems [20]). By leveraging sensory concurrency to create effective feedback which promotes unsupervised 'learn-as-you-do' approaches, users may develop rich new multi-modal skills without direct supervision, helping to remove barriers to uptake of new expressive interfaces.

Finally, the effects presented accentuate the importance of considering study duration and training mechanisms when evaluating the viability of new, SSAD-like interfaces. We distill such findings into guidelines on the design of devices, signals and conditions to promote the creation of expressive interfaces which may be learned through self-guided, productive task engagement. If applied, such principles promote the creation of comparable user studies in literature, and result in improved reliability of studies evaluating the effectiveness of the abstract, dynamic spatiotemporal mappings unique to these interfaces. 


\section{STUDYING VIBROTACTILE SSADS - RELATED WORK}

As described earlier, a number of SSADs have been developed in recent years to support a wide range of user interactions and experiences in different contexts, such as independent navigation using the vOICe [35], FeelSpace [27, 38] or EyeCane [34], aiding communication or hearing via the skin [39, 51], and augmenting awareness during driving [24, 25] or other demanding tasks [6].

The literature demonstrates that participants are able to utilize SSAD device signals through exploration [2], that explicit explanation of device signals and introductory training under some conditions does not necessarily improve performance [14], and that a change in perception [49] may develop with sufficient experience and dedication to the use of the device in context.

SSADs are built on the principles that symbolic information presented by traditional interfaces (especially haptics) is often suitable for simple warning or notification data, but that there may be advantages to encoding more information into dynamic spatio-temporal signals: a sufficiently detailed output signal may allow users to plan and prioritize actions.

Although it is generally accepted that exposure time and device use plays a large part in developing proficiency (and therefore reaching device potential), systematic studies rarely have scope or resources for long-term in-the-wild evaluation of device use, leading to a trend in shorter-term studies and measurements designed to give quick insight into device potential.

In developing such insights, studies face two major challenges: 1) Study metrics should accurately estimate long-term device potential, and 2) Participants should be introduced to the device under conditions that enable and promote proficiency during the limited training time. A strong understanding of how device usage and training contributes to proficiency would allow short-term studies more confidence in testing and training, helping to tackle these challenges.

SSADs manifest in the literature through studies exploring feasibility (evaluating device limitations through psychophysics - see Jones [22] for an introduction), practicability (using methodology derived from contemporary HCI research to compare novel SSAD user performance against unaided performance in specific tasks $[3,6,7,24,25])$, or usability (in-the-wild long-term testing of SSADs with coupled assessments of usability through measurement [37, 47] or interview [48]).

In all studies, accurate metrics and consistent, effective device introduction is critical to the studies' contribution. Below, we address the metrics and training approaches offered by the literature.

\subsection{Study Tasks and Metrics}

Most SSAD studies performed either in-situ or in lab experiments focus on object recognition and/or locomotive tasks. Object recognition tasks often feature in studies involving devices that substitute human vision, producing highly comparable metrics concerning recognition accuracy and speed [1,2]. In short-term studies of this nature, a generalized sensorimotor skill may be differentiated from a rote-learning of responses to symbolic stimuli by the addition of a generalization task, requiring that users identify objects in free response or previously untrained conditions [51]. By contrast, locomotive tasks (such as maze navigation, path completion or obstacle avoidance) are used to measure proficiency in the use of navigational or spatio-temporal SSADs. Such tasks require active attention and comprehension of the SSAD signal for success. It is reasoned that superior proficiency in the interpretation of the device signal results in superior task performance, allowing an estimate of device potential.

Due perhaps to the experiential component of using an SSAD, metrics for success in SSAD studies vary considerably. While finding statistical significance in performance metrics is an important part of determining device or interaction Manuscript submitted to ACM 
effectiveness, quantitative results may also be supported (or refuted) by more qualitative assessment of participant experience and device utilization.

\subsubsection{Quantitative Measures.}

Task Performance: Definite metrics collected using methods derived from conventional HCI experimentation, such as task completion times or object identification accuracy. These metrics provide comparable insights into the utility of a device in a specific context.

Error Rate: Errors in complex task performance (such as the number of collisions measured during maze navigation [14] or attempts in a search and locate task [44]) can provide useful insights into participant awareness and competence, as well as device limitations and fidelity of task information in the SSAD signal.

Brain activity scans, sleep patterns or other biological measurements of learning and perceptual integration: While out of scope for most studies, such measurements have been linked to procedural learning during long term SSAD use [27].

\subsubsection{Qualitative Measures.}

User Experience Data collected through questionnaires capturing Likert/NASA TLX scale data, or interviews, providing sentiment and subjective experience when using SSADs. In a unique study of SSAD utilization, Ward interviews [48] long term users of the vOICe [35]. Weekly supplementary interviews conducted in Konig's long-term evaluation of the FeelSpace Belt [27] supported factor analysis of quantitative questionnaires, bringing increased validity to experiential conclusions.

Process/approaches to the task Exploration of participant mental models, sense-making and task completion/device use strategies can provide insights into how specific devices present user-environment affordances. Lobo [31] specifically analyses route selection in a navigational task for insight into how various devices contribute to users' perception of space in a target approach task using the Enactive Torch.Further, understanding participants' task approach also gives insight into the "what it is like" component of SSAD utilization; a concept at the heart of enactive device design [30] and associated theories of cognition [42].

\subsubsection{Mixed Method Approaches.}

Mixed-method approaches combining user experience data with performance metrics provide insights between condition groups where performance metrics are unable to differentiate, and may lead to longer-term validity of study results.

Nagel [38] and Konig's works [27] are critical literature contributions not only for the uniqueness of the long-term investigations, but also for the confirmatory nature of their qualitative results, which reinforce the suitability of some of their chosen training schemes and metrics (the real-world homing task) in the study, while showing the device and training limitation via others (a virtual navigation task speculated to be too dissimilar to the training environment for the device signal to prove useful).

In the present study, behaviour, process and strategy (through path analysis) are used to assist in differentiating deliberate user approaches from random, mechanical or uncertain efforts at task completion, where performance metrics may not provide a full picture. 


\subsection{Training and Study Duration}

The nature of SSAD training, the duration of SSAD utilization and the feedback provided during use have been identified as key components in the SSAD learning process [4, 44], and to-date there are still many opportunities for systematic studies breaking down the individual contributions of these factors in enabling device proficiency [4].

Here we evaluate how specific findings from short- and long-term studies contribute differently to the literature, but may both benefit from a systematic approach to feedback and evaluation.

Short Term Studies. : Contemporary HCI studies are typically performed over the course of minutes or hours, and are designed to evaluate device effectiveness in specific use cases or contexts.

The Lateral Line [25], Feeling Uncertain [24] and ProximityHat [50] allowed users a familiarization time of 5-15 minutes in the context of a simplified or example use-case, before performing a series of device-dependent navigation tasks. As this length of exposure is significantly too short for the development of new sensorimotor skills, these studies may be relying on a degree of "cross-modal skills transfer"; where participants' familiarity with the task enables quick adaptation to receiving comparable sensory information through a new modality [29].

The original study of the Enactive Torch [14] uses several metrics to offer insight into the long-term potential of SSADs despite the short study duration. Though simple in design (a single-handed device housing a motor which vibrates with intensity proportional to the distance to the object it is pointed at), many users developed "an experience of objects located at a distance in the world" when using the device to navigate a physical maze, suggesting some degree of integration into the participants' perception of space. Despite offering users 40-45 minutes of "training" in a maze navigation task which closely resembled the main study task, the study's mixed-model metrics revealed no difference between trained and untrained users in performance nor sentiment (captured via Likert scale questionnaire). It is unclear whether this was due to the duration or the nature of training.

In these cases, the study goal and associated metrics were focused on device utility with naive users. The application of such measurements alone, combined with the short study durations, highlights specific limitations.

Long-term Studies. : Two long-term systematic studies follow the training, use and evaluation of (specifically) vibrotactile SSADs. The seminal, now well-cited study of the Feelspace Belt [38] evaluates how wearing a vibrotactile belt (which "points" towards magnetic north) during waking hours and simple training tasks for 7 weeks affects participants' navigation abilities. Following training, participants engaged in mixed-method tests which revealed both significant effects in performance (under conditions similar to training) and reported experiential differences in comparison to control participants who did not wear the belt.

Several years later, Konig et. al explore the effects of long-term use of the belt using fMRI and EEG [27], demonstrating that long-term use of the belt (under similar nature and duration to the original study) leads to brain activity typically coincident with procedural learning and navigation. Konig's results show that brain activity indicative of learning effects during SSAD use return to near baseline levels after 4 nights of daily use, suggesting that medium-term exposure to SSADs may prove advantageous over single session experiments.

While both studies reveal interesting details about the effects of long-term SSAD use on perception, it is not clear how the shared duration of 7 weeks was chosen, nor which elements of training and exposure contributed to the development of device proficiency.

Manuscript submitted to ACM 


\subsection{Training Strategies for Sensory Augmentation Devices}

To summarize, "familiarization" typically involves using the SSAD in a free exploration environment where natural senses and device signals are available concurrently, while "training" consists of performing a device- and contextrelevant task, often in combination with sensory deprivation (such as blindfolds). However, the effects of the contrasting sensory approaches remain entangled. A derived approach to designing SSAD training and familiarization mechanisms is required. To date, there are very few systematic studies evaluating such mechanisms $[4,23,28]$.

2.3.1 Implicit vs. Explicit Training. Bertram and Stafford's detailed review on learning of SSAD signals [4] identifies feedback as a minimum requirement for learning of SSADs, and is presented in two distinct forms:

Implicit Feedback - By nature, the signal from an SSAD is itself a form of "implicit" feedback in response to user action. It has been demonstrated since SSAD conception that user control is a necessity for learning; users must be able to observe the effects of their actions on the signal [2]. Changing the signal outwith the context of user action is not sufficient for the development of proficiency.

Explicit Feedback - Additional "explicit" or external feedback may be provided in the context of a goal-oriented task. This may be as simple as the success/failure to complete the task, or more specifically through measures of performance.

"Familiarization" in shorter studies [3,14] and longer-term belt wearing in the FeelSpace studies [27, 38] points towards a general awareness of the importance of time spent with implicit feedback: it is expected that users will develop proficiency simply through exposure to the signal, even when not performing task-specific actions. Equally, most studies seem to agree that "dedicated training” must feature an explicit, task-specific feedback component.

2.3.2 Sensory Modalities as Part of Training. The authors found little experimentation surrounding how implicit feedback via sensory concurrency (i.e. which senses are available during in-situ or training environment device usage) affects the speed and effectiveness of learning to use new SSADs. Of those found, sensory concurrency has been shown to play an important part in learning effectiveness [20, 32, 44].

In both FeelSpace projects' training sessions, participants wearing the belt (and controls not wearing the belt) were asked to make navigation judgments while blindfolded, then permitted to open the blindfold for feedback. Thus, the individual contributions of implicit and explicit feedback during the everyday wearing of the belt are unclear.

Proulx [44] begins an investigation into how the effects of familiarity and training may be de-tangled, assessing the effects of "learning-by-doing" in a 3-week study, finding that participants using an SSAD during unrelated daily activities outperformed those who used it during training and testing only. However, long-term sensory deprivation (blindfolding) did not notably influence performance.

By contrast, in a pretest-practice-posttest study, Lobo [32] showed sensory deprivation (blindfolding) to be more effective than full sensory concurrency (no blindfolding) when learning to use a leg-mounted obstacle detecting SSAD.

Between the measurements of application specific devices and the results of longer-term studies exploring human perception, it is clear that vibrotactile SSADs are capable of enabling rich interactivity; often offering measurable improvements in decision making and allowing planning at a pre-cognitive level. However, as research interest grows, it is critical that participants are introduced to these devices via grounded methodology which allows comparable evaluation of long-term potential.

There remains questions of how combinations of modalities available during SSAD usage affects learning and comprehension of the introduced signal, and how such combinations may be best utilized for more effective feedback. We use a mixed-method approach in a medium-term study to address this in the following presented experimentation. 


\section{STUDYING THE EFFECT OF VISUAL FEEDBACK ON THE COMPREHENSION OF HAPTIC SIGNALS}

In order to investigate how visual feedback affects the comprehension of haptic signals as part of digital navigation tasks, we designed a mixed-method study that combines quantitative measures of performance with qualitative measures of navigation strategy and user experience. Below we provide details of the study design and procedure.

\subsection{Study Design}

Traditional video games often require the navigation of a digital character a form of digital landscape or obstacle path. Frequently, this requires the coordination of hands (via a games controller) and eyes (viewing a screen). In simple games, proficiency is quickly acquired, owing to the versatility of the visual sense and high dexterity of the fingers. Our study is based on a maze navigation task designed to help us explore how various combinations of visual and haptic feedback provided during such a task affect the comprehension and utilization of the haptic signal when all visual feedback is removed. The digital maze navigation task can be considered a proxy for other real-world navigation task scenarios where haptic feedback is used to augment perception.

Our study follows a between-subject design. Each participant performed four sessions of a maze navigation task across four days in a single work-week. In order to compare how different combinations of visual and haptic feedback facilitate the accurate interpretation of haptic feedback over time, participants were divided into four groups - one for each study condition. Participants in each group were introduced to a specific combination of visual and/or spatio-temporal vibrotactile feedback to facilitate their maze navigation. All participants were simply asked to progress through each maze as far as they could. In the first three sessions participants experienced feedback as per the group's condition. Each condition featured a unique combination of visual and/or haptic feedback (detailed below). In the fourth and final session, participants across all groups had to navigate the maze relying on haptic feedback only; direct visual feedback on their position in the maze was removed completely. Accurate and effective interpretation of the haptic signal was essential to successful and effective maze traversal. Participants were not informed that this final session would involve maze navigation using haptic feedback only, to minimize the confounding effects of participants prioritizing learning the haptic signal over performing the task. Before we describe the four study conditions, we provide an overview of the apparatus used as part of the study and how haptic and visual feedback was provided during maze navigation.

\subsection{Study Apparatus}

During each study session, participants were presented with a series of simple, procedurally-generated mazes (see Fig. 1) to navigate using an off-the-shelf official Xbox One game controller. Mazes were presented on a 24" monitor. Below we describe the maze design, how maze navigation was supported through the game controller and how the maze topology was represented in the form of visual and/or haptic feedback.

Maze Design \& Character Navigation. Each maze consisted of a single vertical path of infinite length. Maze paths were procedurally generated by carving a series of square, passable path blocks through a grid of impassable wall blocks. More specifically, two path blocks are placed on top of each other vertically, followed by arranging one to four blocks horizontally to the right or left of the previous blocks, and repeating (see Fig. 1 for two example mazes generated in this way). This enables the automatic generation of different mazes that still bear similarities in terms of topography and difficulty.

In all study conditions and sessions, participants were asked to navigate a space-ship-like digital character forward through these mazes (see Fig. 4.b). Rotation of the digital character in 2D space was supported through the game Manuscript submitted to ACM 


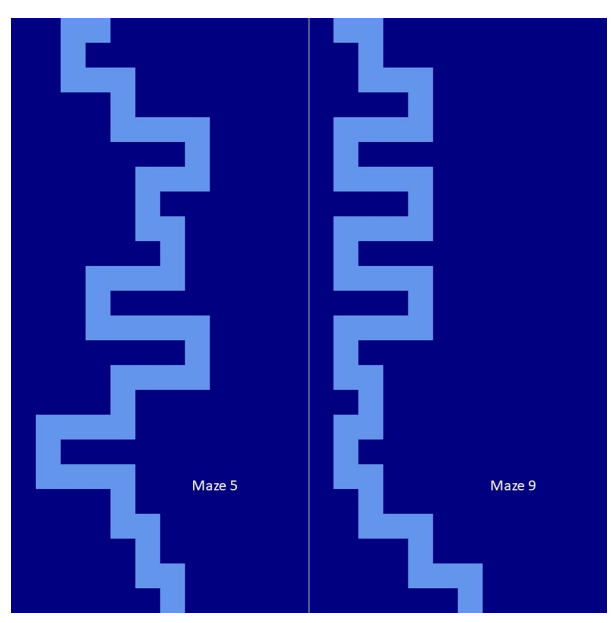

Fig. 1. Two examples of the first 50 blocks of the infinitely-long procedurally-generated mazes used as part of our study: Dark blue areas represent impassable walls; light blue areas represent passable corridors.

controller's primary analogue joy stick. The other buttons of the controller supported moving the character forward in the direction it was facing at a rate of 1 unit per second. Each wall block and corridor had side length 1.5 units. The rotation angle of the character was limited to a range between -90 to +90 degrees ( $9 \mathrm{pm}$ to $3 \mathrm{pm}$ on a circular clock). This enforced a forward movement within the maze, and prevented participants, especially in the haptic-only condition (see below), from navigating backwards by accident. When attempting to continue rotating while at either limit, visual (the walls turning red) or haptic feedback (low frequency two-hand rumble, distinct from the trigger motor feedback) would be provided at a saturation (visual) or intensity (haptic) proportional to the rate at which they were trying to turn (see Fig. 2).

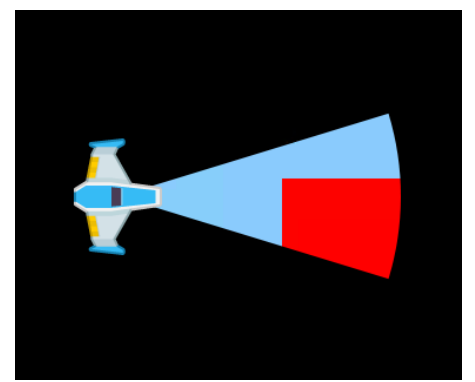

Fig. 2. Illustration of the graphical feedback presented at the digital character rotation limit of +90 degrees when the control stick remains pushed fully to the right.

Game Controller. We decided to use an Xbox One controller for our study as it is a low-cost, off-the-shelf device that enables versatile vibrotactile feedback on a continuous scale. The four embedded Eccentric Rotating Mass (ERM) motors can be independently controlled using the Windows.Gaming.Input namespace in the Universal Windows Platform $\mathrm{API}^{1}$.

$\overline{{ }^{1} \text { Windows UWP }}$ applications API, Windows.Gaming.Input Namespace 


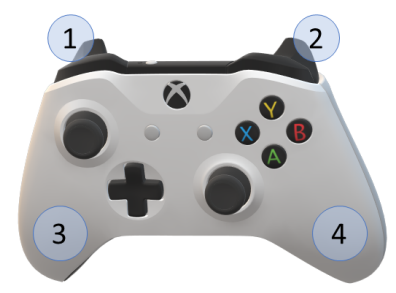

Fig. 3. Microsoft Xbox One wireless controller, showing position of embedded trigger $(1,2)$ and palm $(3,4)$ ERM motors, and button layout.

Two large ERM motors are fixed to the main controller body and located roughly within each palm grip (see Fig 3.3\&4). While these motors operate over different frequency ranges, the resulting vibration when activated is experienced similarly in both hands and, thus, differentiation of signals is difficult. However, two smaller ERM motors are located in each trigger button of the controller (see Fig. 3.1\&2). In terms of the emitted vibrotactile signal, these are well-isolated from each other, and their signal can be easily distinguished and associated with the left and right hands. Their signal also differs from the low-frequency vibration of the largest motor. We therefore decided to use these two trigger motors to produce continuous vibrotactile signals in response to participants' navigation of the digital character in the maze (e.g., direction and proximity to walls, described in detail below), while using the largest palm ERM motor to provide feedback if participants over-stretched the rotation angle of the digital character.

As vibrotactile intensity is typically perceived non-linearly[21, 40], the trigger motors required calibration to support a linear mapping of vibrotactile signal to target information (in our case, the character's distance to a wall). To achieve a linear, symmetrical feedback perceived through left and right controller triggers (see Fig. 3.1\&2), three calibration steps were taken:

1) Each trigger motor's vibrational intensity profile in response to commanded API values was mapped using a microphone in contact with the trigger. The motors were found to be slightly asymmetrical.

2) The range of output signal intensities was restricted to those that both motors were capable of outputting.

3) The API values were parameterized, and re-mapped to a perceptually linear scale according to the Weber-Fechner law [12], using a literature sourced estimated just-noticeable-distance of $12 \%[9,13])$.

Following this calibration, pilot testing with 10 participants who performed a magnitude estimation and an intensity matching task confirmed that the adjusted left and right trigger motor outputs were perceived linearly and symmetrically in response to linear input commands.

Design of Visual \& Haptic Feedback. In the conditions involving visual feedback, the digital character was visible to participants (see Fig. 6.b-d). In addition to the digital character's position in the maze, participants would get visual feedback on walls in close proximity via a visual cone that indicated the peripheral vision angle and viewing distance of the character (see Fig. 4.b and 6.b-d). This cone's angle was 54 degrees in total (see Fig. 4.b). The length of the visual cone (viewing distance) varied across conditions where visual feedback was provided (see description of conditions below).

Figure 5 shows two examples of the visual character navigating the maze in the visual conditions: walls are invisible until they come into view through the digital character's visual cone. The figure illustrates the character approaching a corner in the maze, as indicated through the dark blue space intersecting with the character's visual cone. 
In the conditions involving the haptic feedback mechanism, continuous vibrotactile signals were emitted by the trigger buttons to provide information about distance and direction of walls. This haptic feedback was implemented using two "whiskers" which extend from the character at the same angle as the edges of the visual cone in visual conditions (see Fig. 4.a) and a length of 1 unit. The individual intensity of the left and right trigger motors was determined by the distance to the nearest maze wall within range of the whisker. A whisker touching a wall at their outer periphery resulted in a minimum vibration, while walls touching the character itself were considered a collision, and produced the maximum vibration. If there was no wall within reach of the whisker, the trigger motors did not vibrate.

The maze dimensions were designed so that the character's visual cone and/or whiskers would not touch the maze's walls when centred in a corridor (the default, starting state in each task). Participants would therefore only see or feel a wall if moving forward or turning in a way that would bring them off the maze path centre. That is, one navigation strategy could involve simply minimizing vibration, though the optimal trajectory (and therefore the greatest progress) is achieved by allowing some proximity to walls and corners.
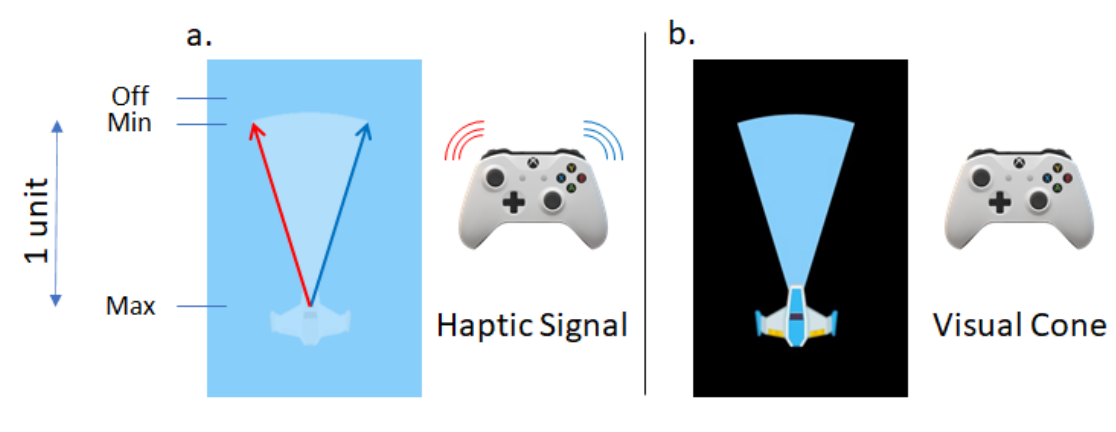

Fig. 4. Haptic signal implementation. Haptic signal (a.) allows independent detection of left (red) and right (blue) walls at a distance identical to full visual cone (b.)
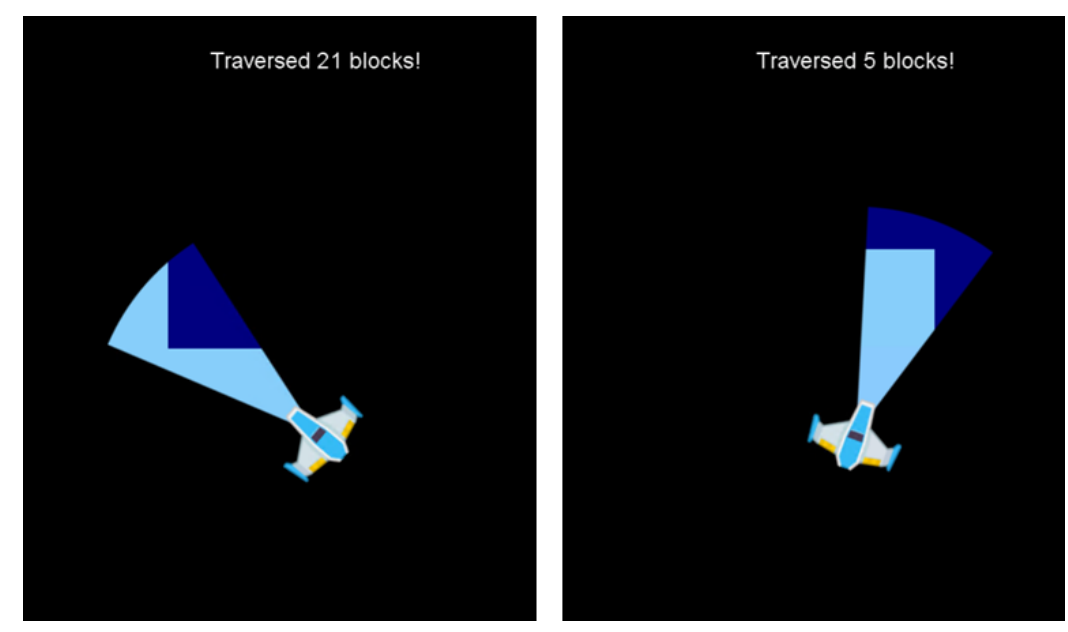

Fig. 5. Examples of maze corners coming into view in the visual condition. 


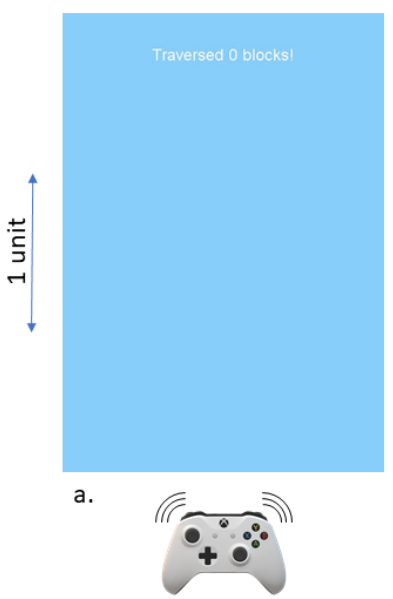

Condition 1 Haptic Only

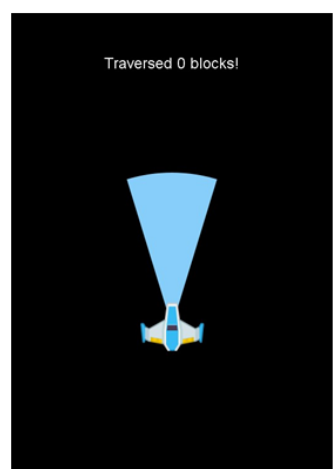

b.

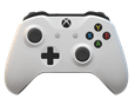

Condition 2

Visual Only

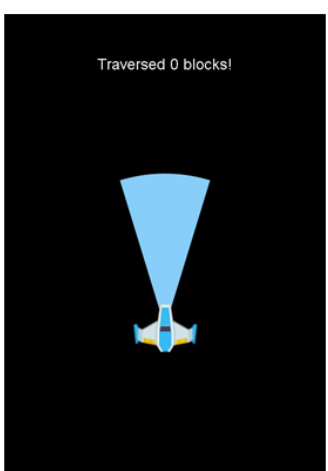

c.

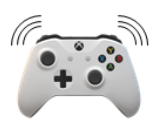

Condition 3

Visual \& Haptic

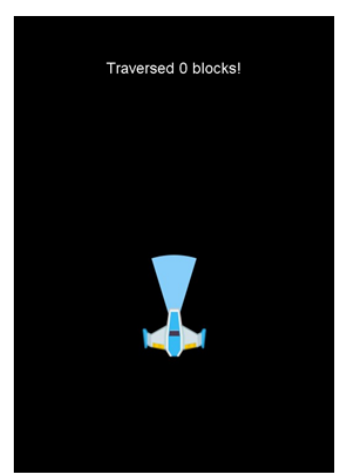

d.

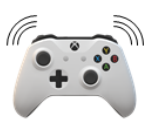

Condition 4

Hybrid

Fig. 6. Illustration of feedback provided as part of the four conditions.

In all conditions, additional visual feedback showed the number of corridor blocks that had been passed, indicating progress through the maze (white text in each of Fig. 6). This visual information constituted the only form of explicit feedback in the study.

\subsection{Study Conditions \& Hypotheses}

The four visual/haptic feedback conditions we tested in this study are designed to resemble some of the familiarization and training mechanisms utilized in the vibrotactile SSAD navigation studies [14, 25, 27, 31, 38] detailed in the related work section. Figure 6 shows an illustration of our four study conditions. We describe each condition and its role as part of our study below.

Condition 1: "Haptic Only". Participants in this group received feedback on their position in the maze via haptic signal only. Neither the maze walls nor the digital character was visible in this condition. In order to progress through the maze, participants had to learn how to interpret the haptic feedback by focusing on the changes of vibrotactile signals received through the game controller, in response to their control inputs. The closer participants navigated the digital character toward a wall, the stronger the vibrotactile signal emitted by the games controller. As described above, the signal included directional information: Figure 4 shows the haptic whiskers used to map wall proximity to the intensity of vibrotactile feedback experienced on the controller's trigger motors. The visual 'sensory deprivation' in this condition resembles the blindfolded training conditions commonly utilized in several other navigation-focused haptic SSAD studies $[6,7,14,31,32]$. Similarly, parallels may be drawn between this condition and the utilization of a novel SSAD by sensorily impaired individuals, whose sensory apparatus may offer little to no overlap with the information presented by the device. Device signals must be learned through task performance and careful attention to explicit feedback.

Manuscript submitted to ACM 
Condition 2: "Visual Only". In this condition, participants saw the virtual character's position and orientation in the maze (see Fig. 6.b) as well as its viewing cone, in which any maze walls present are rendered. The viewing cone was 1 unit long. Haptic feedback was not provided. This visual feedback conveys more information than the haptic feedback in Condition 1: It shows the rotation of the digital character and allows some appreciation for maze corner topography. After navigating the maze under this condition, participants will begin the final session naïve to the meaning of the haptic signal. It is possible that the visual skills gained in the first three sessions may allow participants to tactilely recognize features in the maze in the final session. Such 'cross-modal skills transfer' in the context of SSADs has been explored across several modalities (see Deroy \& Auvray for an overview [10] or Levy-Tzedek for an applied example [29]). Alternatively, a cognitive understanding of the maze structures, controls and shape of the visual cone may allow for fast uptake of the novel haptic signal during navigation.

Condition 3: "Full Visual \& Haptic Feedback". Participants in this condition were provided with haptic and visual feedback simultaneously as they navigated through the maze (see Fig. 6.c). Visual and haptic feedback in this condition combines the feedback mechanisms provided in Conditions 1 and 2: there is a direct correlation between the visual and haptic feedback. As participants approach a wall in the maze, they see the wall coming closer, while experiencing a stronger haptic signal in the controller. Both feedback mechanisms therefore work independently to facilitate participants' navigation through the maze, but, in contrast to Conditions 1 and 2, this condition allows participants to learn how to interpret the haptic signal, based on the visual feedback they receive simultaneously. The effectiveness of such 'sensory concurrency' in learning new feedback signals has been studied in comparison to sensory deprivation [32] and active or user-controlled feedback [20]. This sensory combination is often described as a 'familiarization' mechanism in short-term SSAD studies $[3,6]$.

Condition 4: "Visual \& Haptic Hybrid". In this condition, the length of the cone of vision was reduced to 0.5 units, providing $50 \%$ of the viewing distance compared to Conditions 2 and 3 (see Fig. 6.d). That is, participants could not see as far in the maze as in the two previous conditions. At the same time, participants received the same haptic feedback as in Conditions 1 and 3. Condition 4, thus, provided reduced visual feedback, but information on the maze topology in relation to the digital character was still available through haptic feedback. While participants did not need to pay attention to the haptic feedback to navigate the maze, correct interpretation of the haptic signal while navigating the maze provided an advantage. This Hybrid condition was designed to combine and optimize the beneficial effects of sensory concurrency and sensory deprivation, and was therefore hypothesized to enable most effective learning of the introduced signal (as measured by performance metrics).

\subsection{Participants \& Study Procedure}

We recruited 24 participants (5 males, 19 females) to take part in our study. Participants' ages ranged between 18 and 31 years $\left(M_{\text {age }}=21.4\right.$ years, $S D=3.5$ years $)$. Participants all reported normal or corrected vision. To minimize potential confounding effects of prior experience with navigation tasks in video games and interaction with the physical game controller itself, we only recruited participants who self-identified as having "little to no video gaming experience". Participants were provided with a $£ 20$ book voucher for a local bookstore to compensate them for their time.

We divided participants randomly into four groups-one for each condition, resulting in a 4x6 between-subjects design (four study conditions as described above; six participants per condition group). We decided for a between-subject experiment to eliminate learning effects, which we anticipated would be significant. Each participant attended four separate subsequent study sessions that were spread out over a maximum of five days with a maximum of one session 


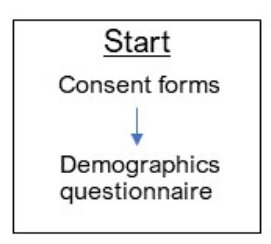

(a)

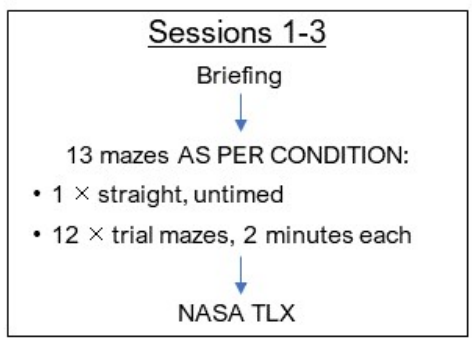

(b)

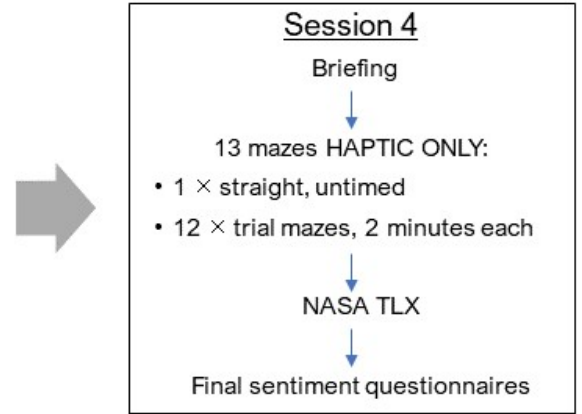

(c)

Fig. 7. Flow diagram showing experimental progression across sessions

per day. This was to ensure that participants were able to sleep after each session-an important component when it comes to the acquisition of procedural task knowledge [27] which the maze navigation task falls under. The gap between each session was never less than 18 hours and no more than 48 hours.

The number of participants and conditions was chosen as a reasonable compromise between allowing the 4-day study to be conducted within a feasible timeframe, and allowing adequate data collection for statistical analysis of repeated measures.

As described above, participants attended four study sessions in total. During the first three sessions they navigated the infinite mazes, making use of the feedback depending on the condition they were assigned to. As part of the fourth and final session, participants across all condition groups were asked to navigate the mazes with only haptic feedback only (identical to Condition 1. See Fig. 7 for an illustration of study sessions).

Before beginning the first session (Fig. 7.a), participants signed the consent form and completed an initial questionnaire to determine basic demographic information (age, reported gender, handedness and any visual impairments). Following this, the first session started (Fig. 7.b).

All sessions began by verbally briefing participants that they would be performing a maze navigation task and that the objective was simply to make as much progress through each maze as possible. Participants were ask to hold the game controller so that their index fingers made contact with the trigger buttons. They were then given time to explore an introductory training maze consisting of a straight corridor of nine blocks to familiarize themselves with the game controller and to ensure that they had understood the task. Participants were asked to navigate to the end of this maze in their own time, to give them the opportunity to explore the functionality of the game controller. The feedback condition during this practice task matched the condition group and session to which participants were assigned.

Participants wore headphones playing brown noise during the experiment to mask the sound of the motors.

They were then given 12 mazes to navigate in a session. 48 mazes were procedurally generated prior to the study as described above; no maze was the same but their level of difficulty was comparable. All participants across conditions and study sessions navigated the same mazes in the same order; 12 mazes per study session. Participants were given two minutes to make as much progress through each maze as they could. Participants were given a 20-second break between each maze. After each study session, participants were asked to complete a NASA TLX survey [18] to coarsely 
capture their perceived efforts, including mental load, temporal load, effort, frustration and a self-estimate of success. The same procedure was repeated across all four study sessions.

In the final Session 4 (Fig. 7.c), participants navigated mazes using haptic feedback only. At the end of the session, participants were asked to complete an additional questionnaire (detailed below) designed to capture their overall experience of the navigation task, considering the two different feedback conditions they had experienced.

\subsection{Data Collection \& Analysis}

As part of this mixed-method study, we collected a range of quantitative and qualitative data to assess participants' maze navigation performance. We focused on their measured performance, their navigation strategies across the four study sessions, and the qualitative experience and perceived difficulty of the tasks.

Quantitative Data. In terms of performance measures, for each maze navigation (trial), we measured the number of blocks participants managed to traverse within two minutes. We also measured the number of times they collided with a maze wall. This quantitative data was analyzed using standard statistical methods (see more details in the results section).

As described above, we also collected data about participants' perceived performance and task effort via a NASA TLX questionnaire and custom questionnaires that focused more on participant sentiment, attention, and intuition during the task through Likert scales (analyzed using standard statistical methods, presented with participant responses in Fig. 17) and open-ended questions, which contributed to the qualitative data collected.

Qualitative Data. In order to investigate participants' navigation strategies, we computationally logged participants' interactions with the digital character for every trial, so we could accurately replicate and analyze participants' traces through each maze. These navigation traces were analyzed visually and qualitatively coded with regard to visual patterns that would provide insights about participants' navigation strategies. After the final session, participants were invited to answer open-ended questions with additional prompt for commentary, to supplement this analysis: "What did you find most enjoyable about the final session (if you found it enjoyable at all). What did you find the most frustrating about the final session? Any final/further comments, thoughts or ideas you would like to communicate?".

There were no issues, errors or missing values detected during data collection and analysis. Trial 0 (the straight corridor training maze) at the start of each session has been excluded from further analysis. Our results are based on the analysis of this quantitative and qualitative data. We describe our findings in the following section. 


\section{RESULTS}

Our study results are presented in three parts. First we report on the findings regarding participants' performance in the different conditions we derived from the quantitative data collected during the study (number of blocks traversed, collisions and a calculated combination of the two). This is followed by an outline of our qualitative findings on maze navigation strategies, in relation to these performance measures. Finally, we report on participants' perceived experience of effort, enjoyability and frustration during the maze navigation under the different conditions, based on our analysis of the post-session surveys and post-study questionnaires.

A summary of participant quantitative data across all sessions is presented for completeness in the Appendix. Data collected during the first three sessions cannot be directly compared with final session data (outlined green) due to differences in task conditions, and will not be further analyzed.

\subsection{Effects of Training Modalities on Performance}

Our analysis of participants' performance with regard to the maze navigation included three aspects. (1) A quantitative analysis of the number of blocks traversed during the provided two minutes per trial in Session 4, (2) an analysis of the number of wall collisions in the context of the number of blocks traversed, and (3) patterns that emerge from these analyses in terms of potential maze navigation strategies.

Number of Blocks Traversed. In order to investigate whether the combination of visual and/or haptic feedback presented during the first three sessions has learning effects when navigating the maze using only haptic feedback, we first analyzed participants' maze navigation performance during Session 4, comparing the number of maze blocks traversed.

Using SPSS, we performed a Generalized Linear Mixed Model (GLMM) [36], in similar fashion to Rossi [45]. This model retains information on trial repetitions and participant-specific variability when estimating marginal means for each condition. We performed this as an analysis of the blocks traversed, with condition as a fixed factor and trial nested as a repeated measurement under participant as a random factor. This test showed a significant effect of condition on blocks traversed $(\mathrm{F}(3,15)=19.394, \mathrm{p}<0.001)$.

Estimated marginal means and associated confidence intervals from the model are displayed in Figure 8. Estimated population means for the number of blocks traversed were 18.5 (Haptic Only), 23.2 (Visual Only), 28.1 (Visual \& Haptic) and 49.0 (Hybrid). Pairwise comparisons (after Bonferroni correction) showed that the Hybrid condition blocks traversed was statistically significantly higher than the other three conditions, but there were no further significant differences.

Overall, our results therefore suggest that the conditions have a significant effect on the number of blocks traversed in the final session, perhaps surprising given the limited number of participants in the study. The Hybrid condition enabled the most blocks to be traversed. The Visual \& Haptic (full sensory concurrency), the Visual (skills transfer), and the Haptic (sensory deprivation) resulted in fewer blocks traversed, though the differences between these conditions were not significant.

Number of Wall Collisions. The number of traversed blocks alone (as described above) is not sufficient to describe participants' maze navigation. For example, some participants may have traversed only a few maze blocks within the given time because they were careful to avoid wall collisions, while others may have traversed few blocks as they were unsure how to progress. Linear regression analysis between blocks and collisions (grouping by condition) showed positive correlations for the haptic $(\mathrm{R} 2=0.39, \mathrm{p}<0.01)$, visual $(\mathrm{R} 2=0.60, \mathrm{p}<0.01)$, and visual \& haptic $(\mathrm{R} 2=0.65$, Manuscript submitted to ACM 


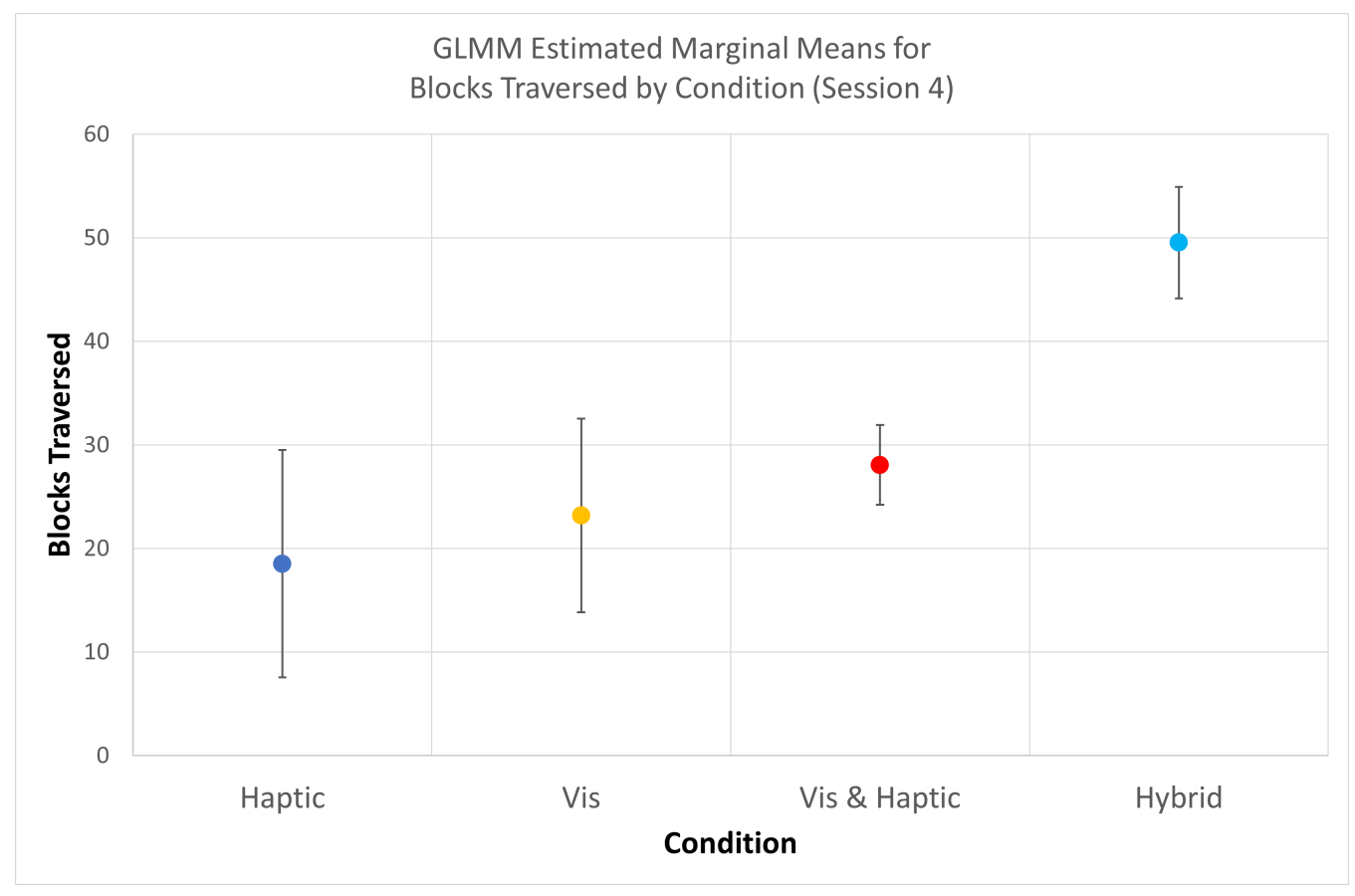

Fig. 8. Estimated Marginal Means and 95\% Confidence Intervals for Number of Blocks Traversed in Session 4. Calculated by GLMM on Blocks with Condition as a fixed factor, and trial nested within participant as a random factor.

$\mathrm{p}<0.01)$ conditions, but a non-significant correlation for the hybrid condition. This confirms that typically, participants who travelled further through the maze on average collided with more walls. As such, no direct quantitative tests were performed on the number of wall collisions alone.

Overall Progress Through the Maze. Similar to Froese [14], a "progress score" was calculated for each trial by dividing the number of collisions by the number of blocks traversed. We then inverted this such that a higher score reflects better performance:

$$
\text { progress }=- \text { collisions } / \text { blocks }
$$

A progress score of 0 indicates perfect performance (no collisions) while progress of less than -1 indicates that participants hit more walls than they traversed blocks.

A GLMM analysis of progress with condition as a fixed factor and trial nested as a repeated measurement under participant as a random factor reached significance $(F(3,22)=5.114, p<0.01)$. Estimated marginal means from the model are visualized in Figure 9. Pairwise comparisons (after Bonferroni correction for multiple comparisons) showed that the Hybrid condition (-0.432) was superior to the Visual condition (-0.704), but that no other pairwise comparisons were significant. Again, the small number of participants in the study must be considered. 


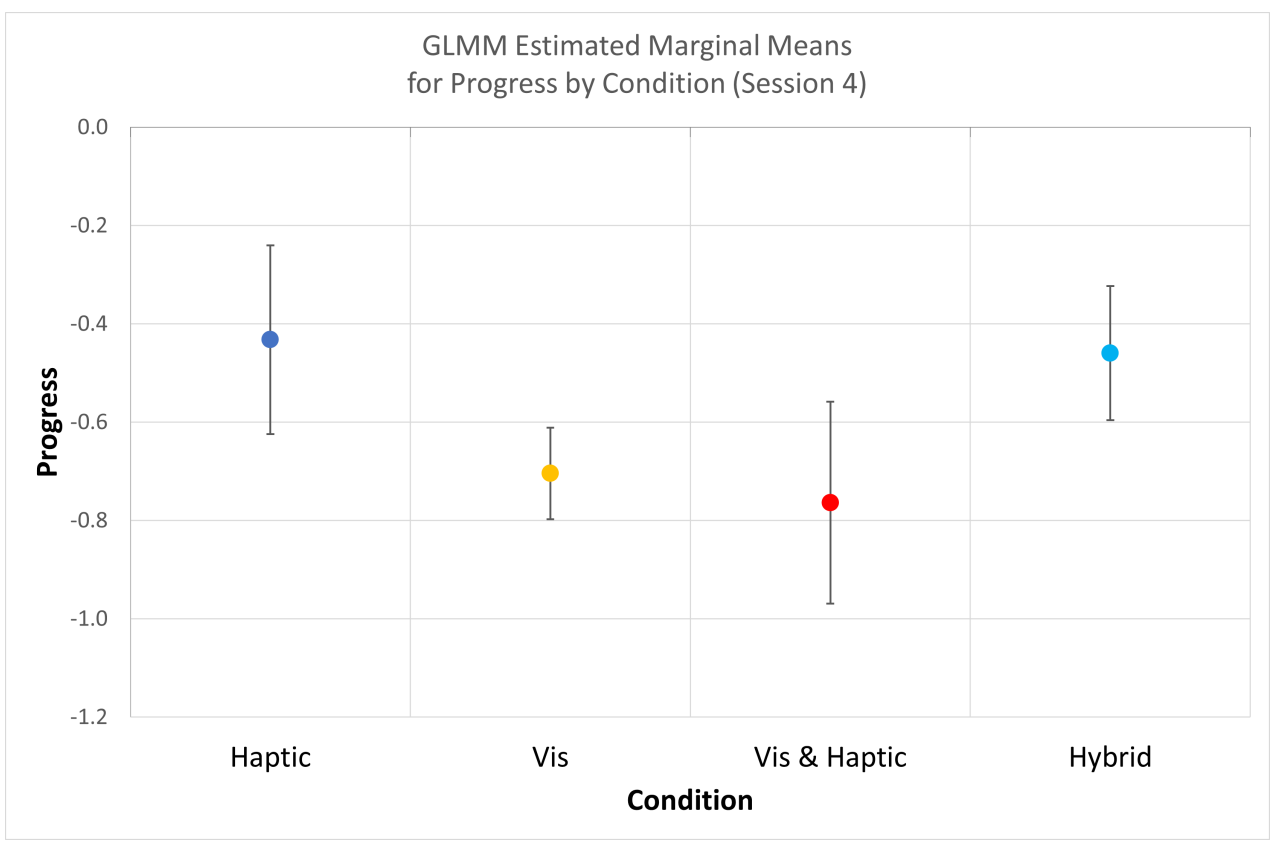

Fig. 9. Estimated Marginal Means and 95\% Confidence Intervals for Progress in Session 4. Calculated by GLMM on Progress with Condition as a fixed factor, and trial nested within participant as a random factor. Progress under the Hybrid condition was found to be statistically significantly higher than the Visual condition.

Individual Participants' Performances Across Conditions. Our quantitative analysis of performance in maze navigation can only provide a high-level, aggregated picture of differences in navigation by condition. This led us to analyze individual participants' performances in Session 4. Figure 10 shows a scatterplot where the y-axis represents "progress" and the x-axis represents "number of blocks traversed". Circles represent individual participants' trials in Session 4 with colour indicating the conditions to which they were assigned (blue $=$ Haptic, orange $=$ Vis, red $=$ Vis \& Haptic, turquoise $=$ Hybrid). The four different quadrants loosely point to different maze navigation strategies in terms of performance:

- Quadrant 1 (bottom left): Few blocks, low progress scores (i.e., high number of wall collisions).

- Quadrant 2 (bottom right): Traversed many blocks, but low progress as they collided with walls often. Could be characterized as reckless-navigation was fast, rather than careful.

- Quadrant 3 (top left): Traversed few blocks, but received a high progress score-very few wall collisions. Likely cautious when navigating the maze. More focused on staying on the path than navigating the maze quickly.

- Quadrant 4 (top right): Traversed many blocks and also received a high progress score. These trials demonstrate efficient navigation strategies.

These quadrants loosely reflect the quantitative differences between conditions. For example, participants in the Haptic condition predominantly fall into Quadrant 3 (top-left; blue circles) representing relatively low scores in term of blocks traversed, but with good progress scores. Participants from the Hybrid condition are largely present in Quadrant 4 (top right; turquoise circles) representing good performance scores. Participants in the Visual (orange) and Visual \& Haptic (red) conditions largely fall into Quadrant 1 (bottom left). 


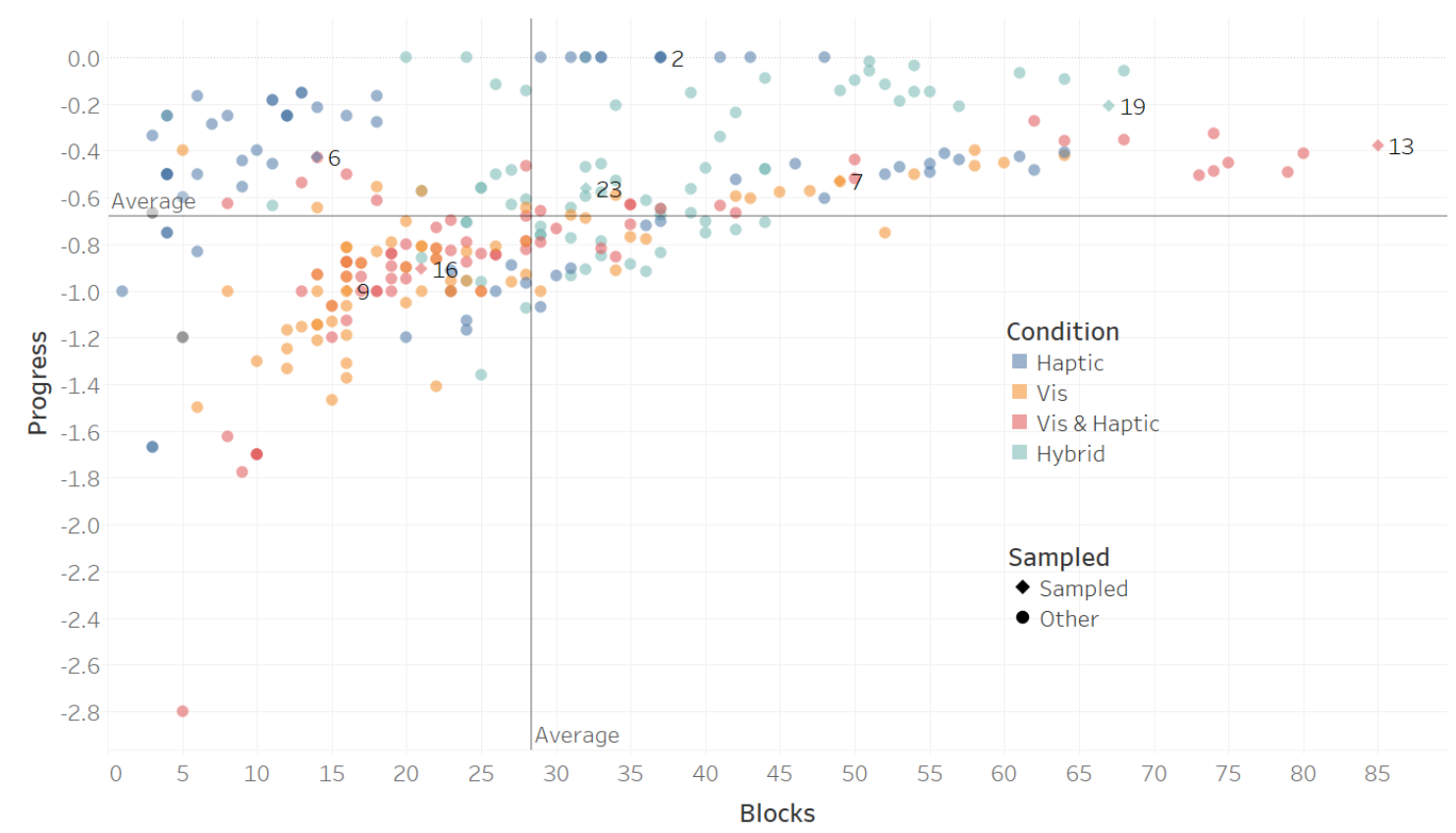

Fig. 10. Session 4 Progress vs. Blocks. This scatterplot shows the number of blocks traversed ( $x$-axis) vs. the amount of progress made (y-axis) in Session 4 (all trials navigated in Session 4, using haptic feedback only). "Progress" is calculated as -collisions/blocks. Each mark represents a single maze navigation (trial), coloured by condition.

The scatterplot also indicates a wide spread of performances with several outliers where some participants outperformed the average of their condition group. For example, participant P2 (Haptic condition), compared to other participants in this group, performed extremely well; their trials can be found in Quadrant 4 (top right). Similarly, the trials by P7 (Visual condition) and P13 (Vis \& Haptic) -all in Quadrant 4 (top right)-suggest a higher performance compared to the those trials by fellow participants in the respective group conditions. These findings led us to conduct a qualitative analysis of selected participants' maze navigation strategies as described below.

\subsection{Maze Navigation Strategies}

Our qualitative analysis of maze navigation strategies focuses on individual participants who seemed to have outperformed fellow group members in their respective conditions. These participants were identified through the scatterplot as shown in Figure 10. We also identified and selected sample participants from each condition that seemed to represent more typical performance score as reflected by the quadrants of the scatterplot. We then compiled the final maze traces of these identified participants and conducted a qualitative visual analysis on the first few blocks to identify different maze navigation strategies, in terms of utilization of the haptic feedback. We compare the traces across conditions to see whether the conditions corresponded with particular methods for signal interpretation and maze navigation strategy.

Note that in the following presented maze trace visualizations (Figs. 11 to 16 ) only the first $\sim 20$ blocks of each (infinite) maze are shown for conciseness. 


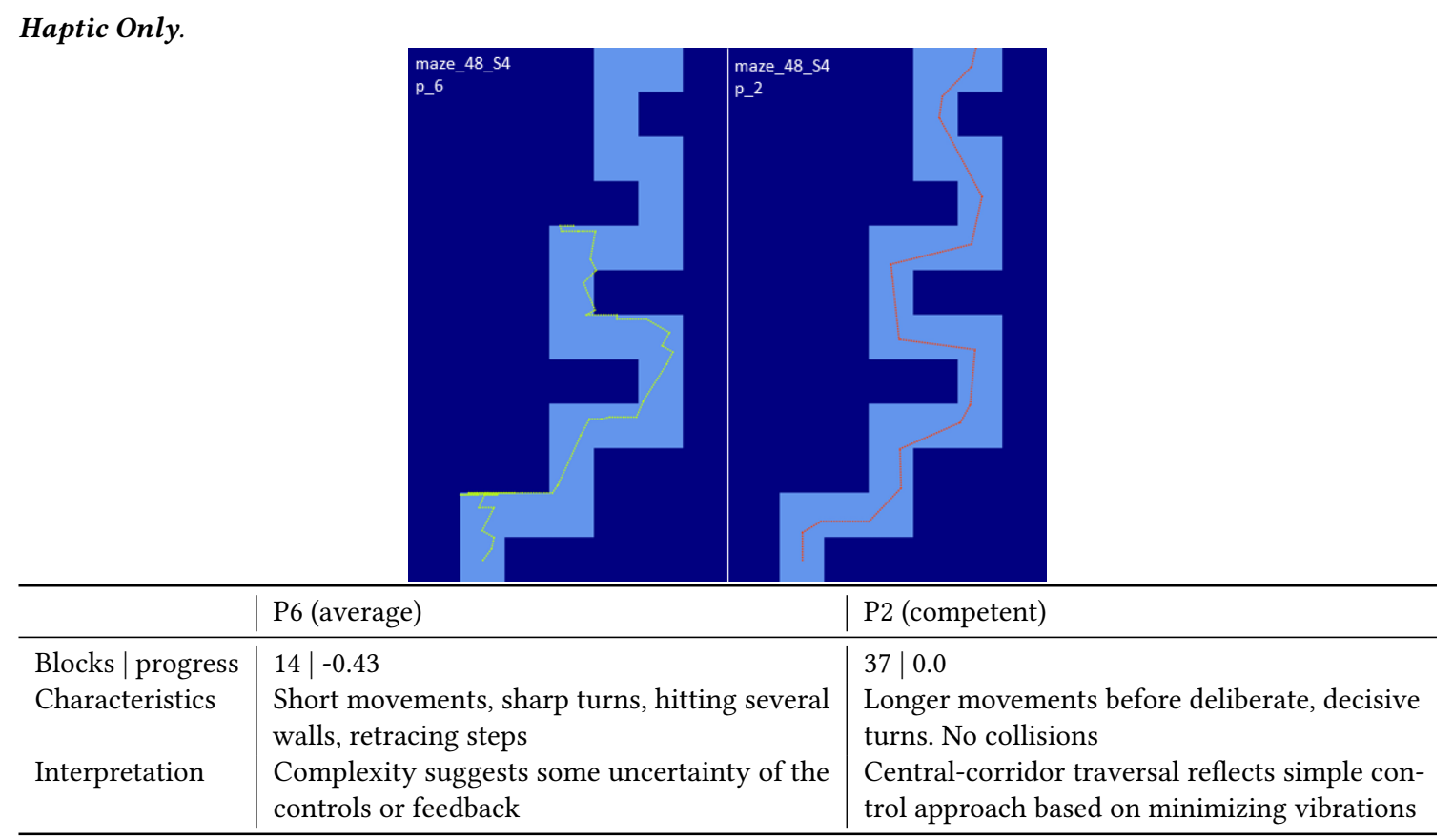

Fig. 11. Comparison of P6 and P2 Session 4, trial 12 traces - Haptic Only

\section{Visual Only.}

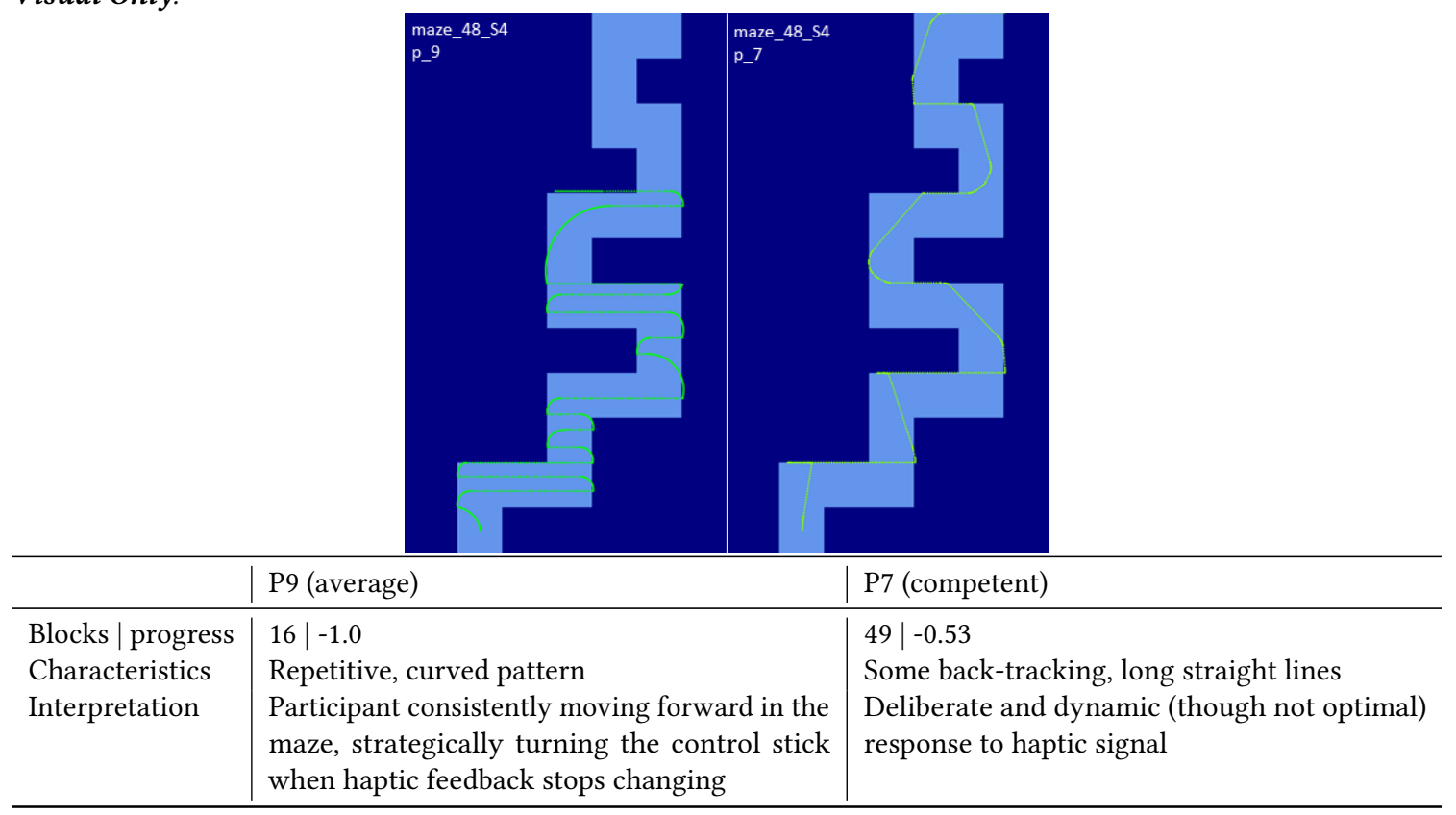

Fig. 12. Comparison of P9 and P7 Session 4, trial 12 traces - Visual Only 


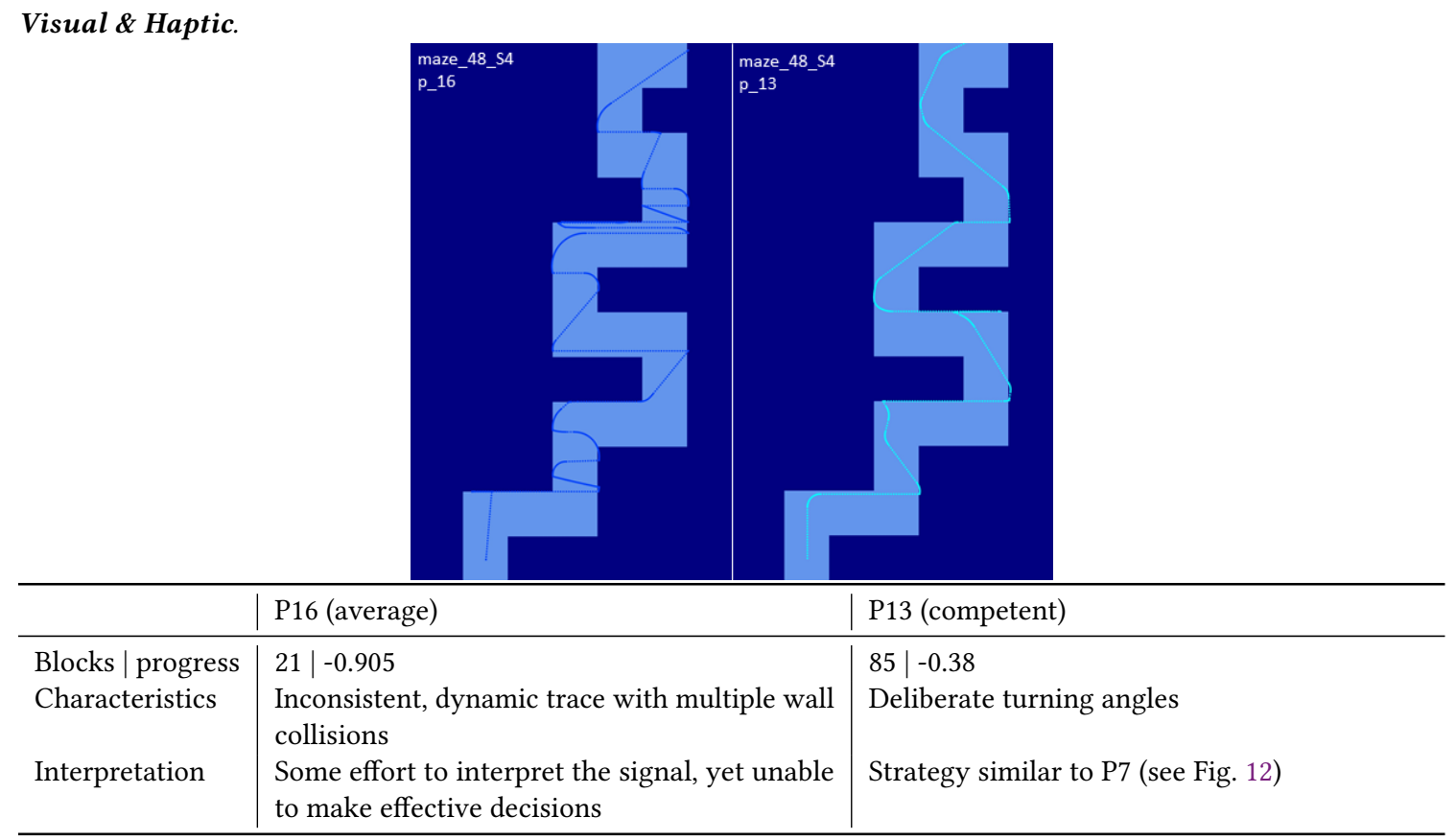

Fig. 13. Comparison of P16 and P13 Session 4, trial 12 traces - Visual \& Haptic

\section{Hybrid.}

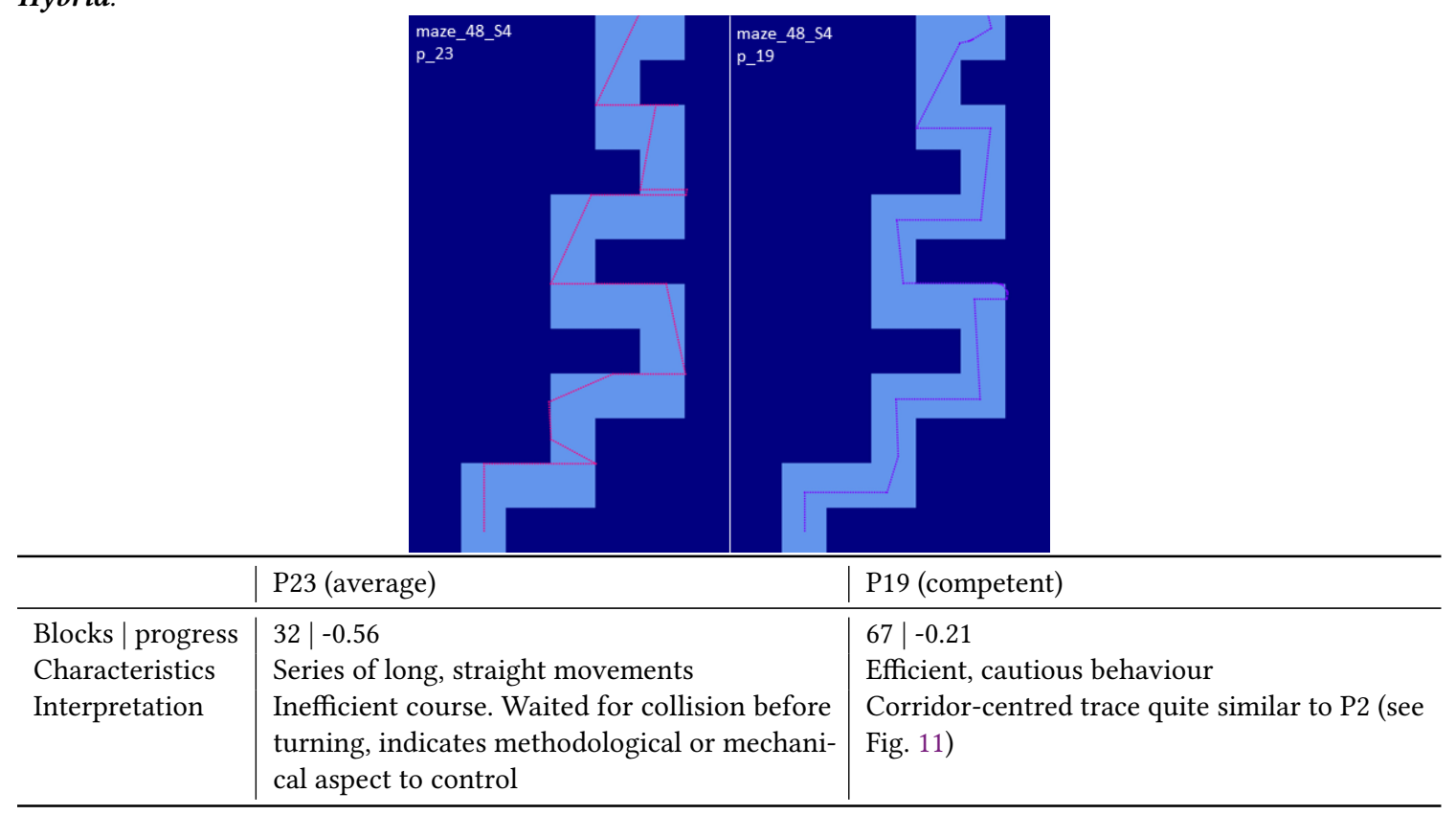

Fig. 14. Comparison of P23 and P19 Session 4, trial 12 traces - Hybrid 
4.2.1 Correlation of traces with Performance Metrics. Here, we compare the traces in Figs. 11, 12, 13 and , 14 with their coordinates on the scatter chart 10 in order to evaluate the effectiveness of the blocks traversed and progress metrics in reflecting maze traversal competency, thus clarifying the importance of the statistical analysis.

Participants 2, 7, 13, 19 and 23 traversed more than the average number of blocks, suggesting that all conditions enable participants to develop the skills required for fast maze traversal. However, the differences between the estimated marginal means across conditions suggests that the condition had an effect on how quickly participants were able to traverse the maze, with participants in the Hybrid condition traversing fastest. Block count decreased across the Vis\&Haptic, the Visual Only, and the Haptic Only conditions respectively.

The participants with the lowest progress scores (participants 9 and 16) display a non-dynamic, repeating or mechanical behaviour in Session 4. Conversely, the other traces show signs of deliberate action, indicating that the progress score is a useful metric for deliberate haptic signal utilization. In combination with the statistically significant differences in progress scores across conditions derived in section 4.1, we can conclude that the Hybrid and Haptic Only conditions were more likely to teach participants to proceed cautiously, while the Visual condition encouraged more reckless traversal of the maze in the final session. The Vis\&Haptic condition resulted in the lowest estimated progress score, but was not statistically significant to other conditions due to wide participant variance, suggesting that the training condition was highly subjective.

As mentioned above, Quadrant 310 predominantly featured participants from the Haptic condition, three of whom never traversed more than 20 blocks in any session, despite consistently achieving a progress score $>-0.5$. This would suggest that the Haptic condition, while effective at teaching participants to avoid walls and navigate the maze deliberately, did not guarantee that participants would become proficient at traversing blocks. This behaviour is reflected in the final trace of P6 (Fig.11).

4.2.2 Individual Strategy Shifts. Most participants' data were clustered in small areas for each session, reflecting a degree of consistency in strategy. However, some participants display step changes or a wide distribution of performance metrics throughout the final session. We were interested to know whether such performance differences may be explained through changes in strategy, and whether such strategies could be identified and differentiated through analysis of the maze traces. Trial-by-trial examination of the data identified two participants with anomalous distributions of blocks vs progress in session 4 :

P7 exhibited significant differences in performance across session 4, achieving 28 blocks at progress -0.93 in trial 2 (maze 38), but 64 blocks at progress -0.43 in Trial 4 (maze 40) as shown in Fig. 15. The large differences in block and progress values are confirmed by clear differences in strategy. Maze 38 (Fig. 15.left) shows a signature curvy side to side pattern, produced when systematically alternating between turning left and then right while holding the advance button. By contrast, Maze 40 (Fig. 15.right) shows more deliberate choice of trajectory and results in a much higher block traversal rate and progress score.

P24's session 4 marks were clustered in two distinct bands, one at Progress - 0.6 and one $>-0.1$, both with traversed block counts of 20-40 blocks. Fig. 16.left shows the traces with progress $>-0.1$, reflecting deliberate and nuanced decision making and good wall avoidance typical of participants in the Hybrid condition. However, Fig. 16.right shows more erratic movement and less regard for wall collisions.

4.2.3 Implications of Quantitative Metrics and Maze Traces. Addressing the performance metrics and maze traces, we can conclude that the Hybrid condition enabled quick yet deliberate maze navigation, the Visual and Vis\&Haptic 


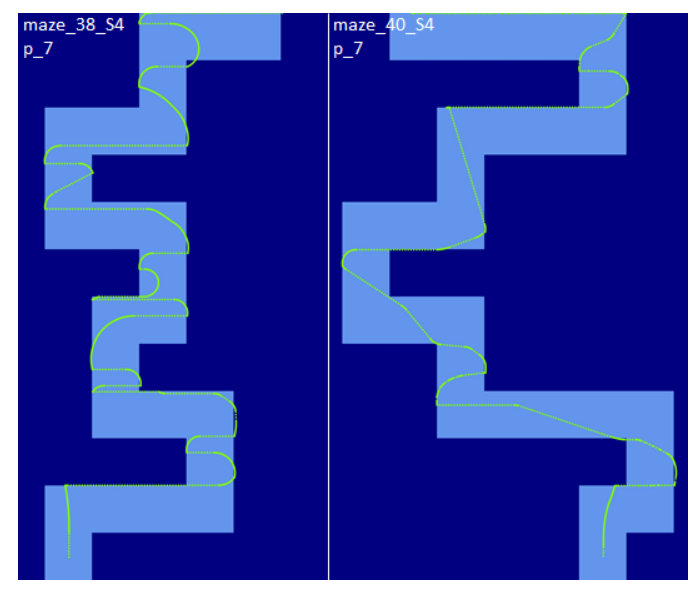

Fig. 15. Participant 7's traces through mazes 38 and 40 (in session 4).

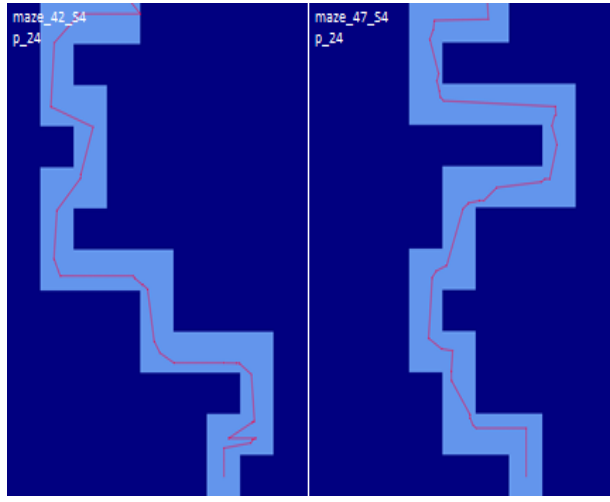

(a) progress $>-0.1$

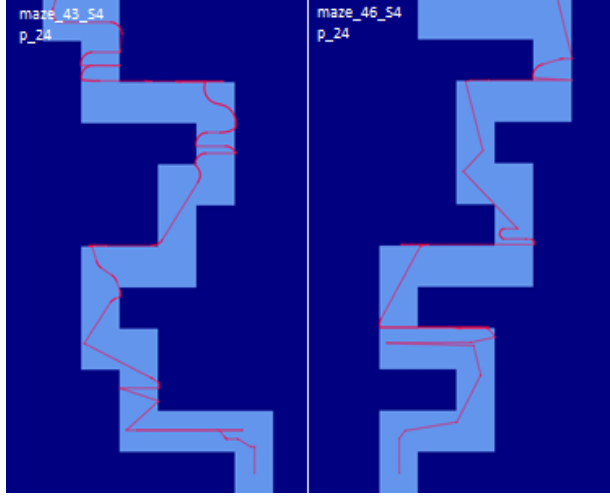

(b) progress -0.6

Fig. 16. Shows Participant 24's high progress traces through mazes 42 and 47 (left) and low progress traces through mazes 43 and 46 (right). Two distinct strategies are employed, resulting in very different progress scores despite similar block count.

conditions promoted moderate speed but more reckless traversal, and the Haptic Only condition encouraged deliberate performance of the task but did not guarantee quick maze traversal.

\subsection{Likert Responses}

At the end of the study, participants were invited to complete a questionnaire designed to capture sentiment and understanding of the game task. The questions and participant responses are visualised in Fig. 17.

A Kruskal-Wallis test comparing the Likert responses across conditions indicated that the only question showing significant difference between conditions was "I felt my performance improved across the sessions". The sample size is small (6 data points per condition) so achieving statistical significance is generally not viable. However, overall trends in sentiment correlate with the quantitative results, so are included here for completeness. 


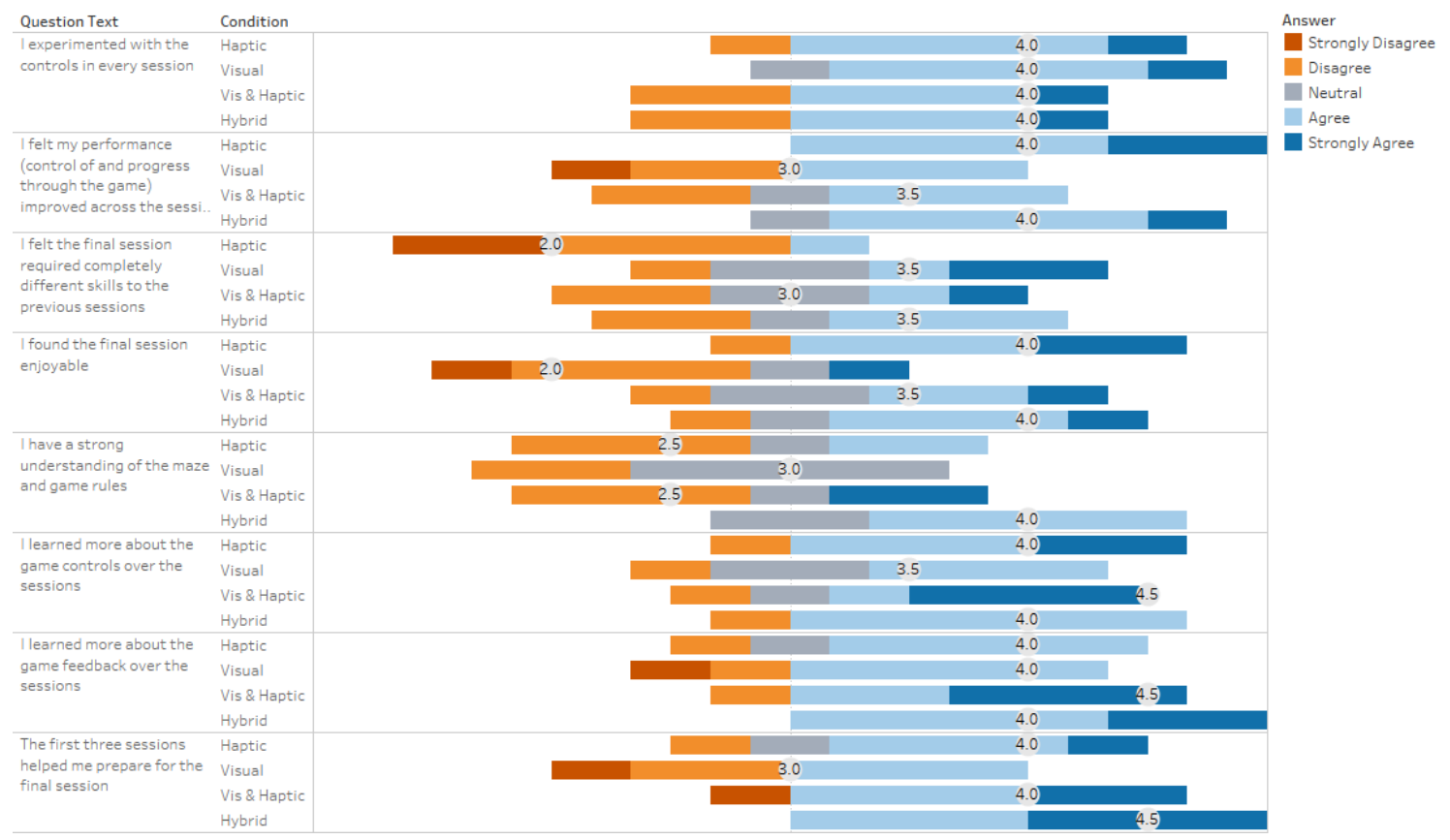

Fig. 17. Shows distribution of responses on diverging stacked bar chart, and overall median response (grey circle).

The greatest discrepancies grouped by condition were in response to the following statements:

"I felt my performance improved across the sessions": Some participants in the Visual and Visual\&Haptic conditions disagreed with this statement. Participants in the Haptic and Hybrid conditions either agreed or were neutral.

"I found the final session enjoyable": Participants in the Visual condition seemed not to enjoy the final session. Participants in the other categories were largely neutral or positive about the final session.

"I have a strong understanding of the maze and game rules": Participants in the Hybrid condition felt on average most confident in their understanding of the game rules and environment, though two participants in the Visual\&Haptic condition strongly agreed with the statement.

"The first three sessions helped me prepare for the final session": The Visual condition left participants feeling that the first three sessions were not helpful in preparing for the final session. All participants in the Hybrid condition agreed or strongly agreed that the first three sessions were helpful.

\subsection{NASA TLX}

We binned the results from the NASA TLX scale into seven equally distributed intervals from "Very High" to "Very Low" and present the results for each index (split by condition) in Figure 18. Similarly, summing scores across participants for each category in each session allowed a comparison of overall scores as seen in Fig. 19. As expected, scores for the Haptic condition decreased gradually across the four sessions. Scores in all categories for all other conditions increased from Session 3 to Session 4, though as with the Likert questionnaire results, the small sample size of TLX data rends achieving statistical significance unfeasible, so no quantitative tests were performed.

Manuscript submitted to ACM 


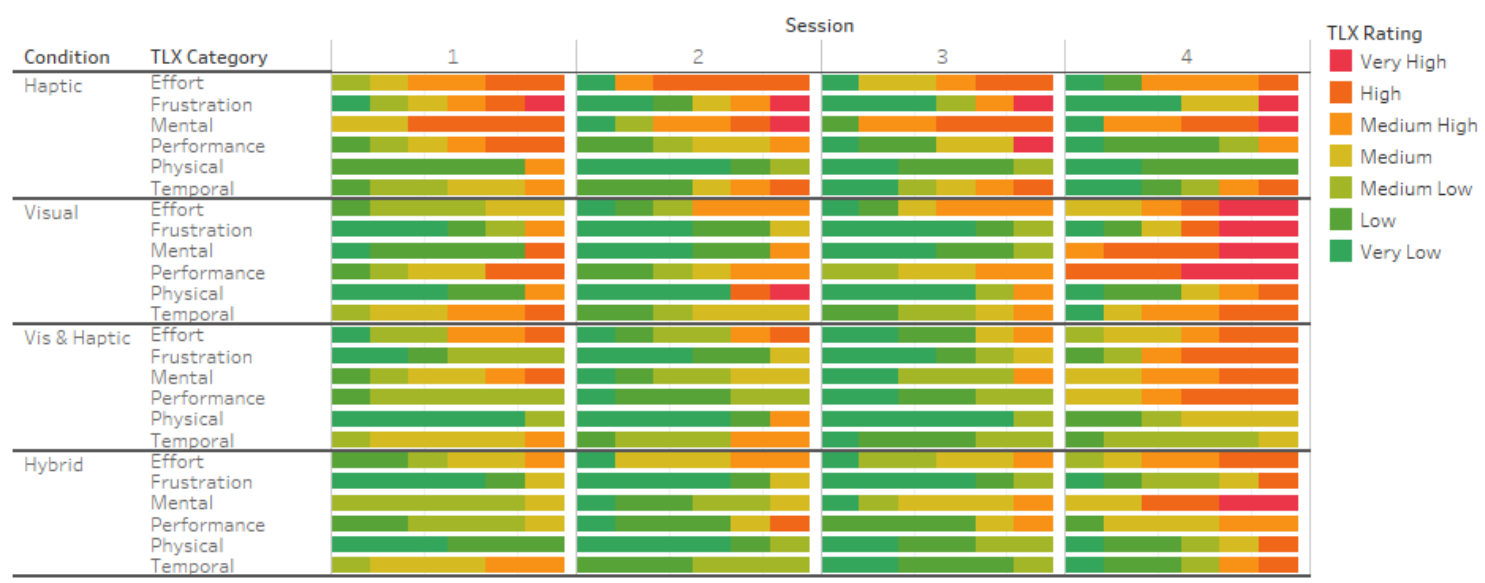

Fig. 18. NASA TLX results showing proportion of participants from each condition responding in each binned measurement group. Greater areas of red show increased load in the corresponding index.

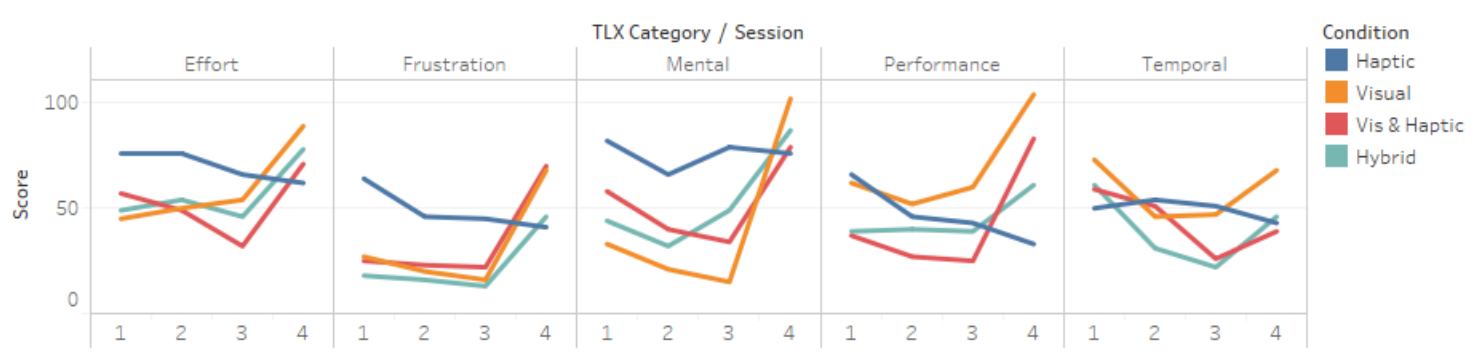

Fig. 19. Sum of NASA TLX scores across all participants, split by session, condition and category.

\subsection{Open Questionnaire Responses}

Through the questionnaire presented at the end of the study, participants were invited to comment on their experience during the final session, some insights of which we summarize here:

"What did you find most enjoyable about the final session (if you found it enjoyable at all)? What did you find the most frustrating about the final session?" From the Haptic condition, P2 described the final session as boring and repetitive, while P1, P3 \& P5 specifically reported enjoying the exploration and discovery process as enjoyable. P1, P4 \& P6 reported frustration from uncertainty of the controls, and P5 \& P3 from being rushed. In the Visual condition, P10, P11 \& P12 described the exploration and novelty of the haptic signal as the most enjoyable aspect, while all participants reported that the lack of progress and not understanding the haptic feedback was frustrating. P13, P14, P17 \& P18 in the Visual \& Haptic condition reported the challenge and attention required in the final session as being the most enjoyable aspect. P13, P14 \& P17 said that getting stuck was the most frustrating part of the session, while the other three reported frustration at not being able to see the vision cone. Participants in the Hybrid condition reported seeing progress (P20 \& P21), engaging in skilful action (P22 \& P23) and trying to beat the scores they had achieved in previous sessions (P19) as the most enjoyable parts of the final session. P19, P20, P22 \&P24 reported being frustrated by 'getting stuck', while P21 suggested that losing the visual cue was most frustrating. 


\section{DISCUSSION}

Our conditions and study design aimed to address two research questions. (1) Does the nature of sensory concurrency (sensory deprivation, cross-modal skills transfer, or full concurrent feedback) presented to participants in the first three study sessions affect performance, strategy and user experience when the visual feedback is removed (Session 4)? If so, how? (2) In light of our hypothesis, what potential do "hybrid" mechanisms, conveying valuable haptic information while retaining confirmatory visual feedback, have in comparison to single modalities?

Below we discuss our findings in the light of these research questions, with reference to the literature.

\subsection{Maze Navigation Strategies Using Haptic Feedback}

As outlined in the previous section, not all participants were able to demonstrate effective maze navigation strategies during the haptic-only Session 4. However, several participants traversed significantly more blocks than others, colliding with few walls (Fig.14.Right) or none at all (Fig.11.Right), demonstrating that deliberate, effective navigation of the virtual maze using haptic feedback alone is indeed possible.

5.1.1 Sensory Concurrency and Maze Navigation. Bertram's review [4] identified 'training/session duration', 'feedback' and 'application similarity' as key influences in learning to use new SSAD signals. By controlling for training session/duration and application similarity, we isolate feedback as the key variable in the comparison of final session haptic-only performance, and thus through demonstration of significant differences in block traversal and progress, conclude that the information available in the visual channel during the first three sessions has a profound effect on haptic-only maze navigation.

However, contrary to Lobo's findings [32], where Lobo observed participants performed better following training with a blindfold on than without, we observe that full sensory deprivation (condition 1, Haptic Only) may be a less effective learning environment than providing concurrent visual information (condition 3, Visual \& Haptic), or even skills transfer (condition 2, Visual Only). The primary differences between our experiment and Lobo's are that in our experiment, participants in the haptic-only condition (visual sensory deprivation) were not at any point provided with an explanation or visualization of the task, and all other participants were not informed about the haptic-only final session. In Lobo's pretest-practice-posttest design, participants were aware of both task context, and the upcoming haptic-only testing session. One explanation for the difference in observed effects may be task comprehension- without the additional context of the visual feedback, participants in our haptic-only condition seemed to find learning difficult, consistently reporting high mental load in the Nasa TLX. This difficulty is apparent in the data visualized in the Appendix. Participants in the Haptic Only condition continued to improve slowly well into Session 4, while nearly all other conditions' participants had reached a performance plateau in earlier sessions.

Participants in the Visual Only condition were able to traverse more blocks in Session 4 than those in the Haptic Only condition, suggesting that visualization of the task contributes significantly to comprehension. Nevertheless, this result is somewhat surprising, as participants in the visual-only condition had no prior exposure to the haptic signal until they encountered it in Session 4. The degree of skill shown during the final session by participants in the Visual Only condition demonstrates the expected competence of untrained participants performing a familiar task using an unfamiliar SSAD. Participants in the Visual condition performed weakly in the haptic-only task (relative to those in the Hybrid and Visual \& Haptic conditions); so while it is reasonable that asking users to utilize a novel SSAD to perform a familiar task could give a useful indication of the intuitiveness of the device, we can be confident that this is not an effective mechanism for enabling or demonstrating the long term potential or effects of proficient use of the SSAD.

Manuscript submitted to ACM 
Participants who performed the first three sessions under the Visual Only condition in general did not enjoy the final session, also reporting consistently high Nasa TLX scores across load index categories (Fig. 18). However of the other participants, as two thirds said they enjoyed the final session, this suggests that such a control scheme or mechanism may be of interest in future gaming or entertainment applications. As participants in the Hybrid condition unanimously agreed or strongly agreed that "The first three sessions helped me prepare for the final session", this mechanism could also be a viable method for introducing to novel haptic control systems in such applications.

5.1.2 Hybrid Sensory Concurrency. As concluded in Section 4, the Hybrid condition was the most successful condition at preparing users for quick maze traversal, as indicated by the overall number of blocks traversed. Furthermore, it was on par with the Haptic Only condition (and perhaps the Visual\&Haptic condition) in enabling and encouraging deliberate use of the haptic signal as indicated by the high overall progress scores. This correlates with the response that all six participants in the Hybrid condition agreed or strongly agreed that "The first three sessions helped me prepare for the final session" (Fig. 17. These results suggest that the Hybrid case was indeed effective as a learning environment: to answer our hypothesis (Section 1), there may be an optimal level of sensory concurrency which enables sensory integration at a rate and depth superior to both sensory deprivation and full sensory concurrency.

\subsection{Feedback Mechanisms in SSAD Studies}

In parallel with Lobo [32], we remark that our findings align with the Guidance Hypothesis [20], which suggests that the more learners rely on some type of feedback during practice, the more they come to depend on that feedback. By reducing the information in the visual channel as applied in the "Hybrid" condition of our study during Sessions 1 to 3 , participants may have been encouraged and incentivized to pay attention to and learn the haptic signal, which seems to have helped them in Session 4 in the haptic-only setting.

Similarly, studies by Huet [20] have found user-controlled confirmatory feedback (where users are able to request easily comprehensible task feedback at will) to be more effective than scheduled, incomplete or ambiguous feedback. Based on our study findings, we argue that by retaining an element of the visual, the Hybrid condition provides confirmatory feedback through sensory concurrency which seems to be beneficial for learning how to interpret haptic feedback. Our findings may suggest that the Hybrid approach combines aspects of both user-controlled confirmatory feedback and the Guidance Hypothesis; dissuading users from developing a disadvantageous reliance on the visual channel during the task, while providing easily comprehensible feedback when users approach walls.

The simple nature of our hybrid approach has some further advantages over user-controlled confirmatory feedback. Firstly, providing a minimalist yet familiar sensory signal alongside a useful new sensory augmentation signal allows the augmentation to be learned without impairment of usual task performance. Further, being a passive condition which does not require users to actively request additional information, it is simpler both in terms of implementation and utilization. We also note that such a hybridized concurrency may be introduced to users in stages: future work may consider the gradual reduction of visual signal in order to guide users towards greater dependence on the haptic signal.

Given the advantages that task context brings to the integration of the haptic signal, we could conceive that further explicit feedback and guidance on the nature and design of the haptic signal, even a simple explanation of how the whiskers were implemented, may further enhance the effectiveness of the Hybrid approach to sensory feedback. Equally, it is arguable that explicit feedback such as the number of blocks traversed may not be required at all in real-world goal-driven tasks with user-defined metrics of success (such as safe, efficient driving or remote communication). 


\subsection{Application of the Hybrid Learning Condition in SSAD Studies}

Conditions comparable to the Visual \& Haptic condition of our study are often utilized in SSAD studies through a 'familiarization' process as described in section 2.2. In short term studies, participants are typically briefly exposed to the novel SSAD signal with full sensory concurrency (uninhibited visual and proprioceptive cues) before performing the task using the novel signal only (e.g. [50]). Similarly, both long-term FeelSpace belt studies [27, 38] have utilized this mechanism: Participants were asked to wear the FeelSpace belt during waking hours for several weeks and received belt information alongside visual, auditory and proprioceptive navigational cues, before the degree of signal understanding was tested. However, as in our Visual \& Haptic condition, the haptic cues in the short term and FeelSpace studies are largely superfluous to the spatiotemporal information still available in visual (and proprioceptive) cues, which may limit development of proficiency as it did in the present study. Implementing the principles of the Hybrid condition in such studies may encourage more effective signal utilization, improving study reliability and revealing longer-term potential of the SSADs.

The Hybrid condition was designed under the principle that the novel modality must form a key part in decision making: providing highly task-relevant information unavailable in other senses, while retaining confirmatory information in existing senses. These principles may be applied to SSAD studies in several ways; for example, by partially depriving some sensory cues such that the novel SSAD signal is more relevant, or by designing training tasks which may be completed without the SSAD, but where an understanding of the haptic signal provides an advantage.

Revisiting Kruger's Lateral Line study [25], we note that such Hybrid principles are present by nature of the system design, potentially explaining why successful signal utilization was demonstrated by participants despite the short study duration and lack of dedicated training: At will, users of Kruger's system are able to refer to visual feedback present in the rear-view mirrors, receiving information concurrent with the novel haptic feedback. While the haptic feedback is not necessary for task completion, users able to utilize it gain a decisive advantage, thus the user self-guides towards proficiency in comprehending the novel signal.

\subsection{Limitations and Open Questions}

As with many studies in the field of SSADs, the number of participants recruited for investigation limits the conclusions that can be drawn from observed phenomena, particularly limiting the power of statistical tests. Applying a rigorous methodology and gathering multiple data points per participant go some way to combating this, as does conducting the study over a medium term of 4 days. While the consideration of participant as a random variable in the analysis methodology offers robustness against this, expanding on this study with a larger sample size may assist in clarifying the contributions of individual differences to the effectiveness of each condition.

In this study, participants were kept naïve to the haptic-only nature of the final session, in order to reduce the confounding effect of directed focus on the haptic signal. Given the aforementioned contradictory findings of our study against Lobo's[31], addressing the role of deliberated attention on the learning process in future work may assist in explaining why this is the case.

Finally, we note that the Xbox One controller itself has also proven a suitable tool for the research of vibrotactile signals, and similar game or VR controllers (such as the Oculus Rift Touch [41] or HTC Vive Cosmos [19]) may also be useful off-the-shelf for exploration of vibrotactile navigation systems and similar SSADs.

Manuscript submitted to ACM 


\section{CONCLUSIONS}

In the present study, we have shown that the nature of haptic and visual sensory concurrency presented to users during a virtual navigation task has a strong effect on how users perform when the visual element is removed. Users initially presented with haptic feedback alongside a limited visual signal respond and perform better than users initially presented with haptic feedback only, visual feedback only, or concurrent haptic and visual feedback.

In enabling users from all conditions to navigate the virtual maze using haptic feedback alone, we have also demonstrated the effective use of the Microsoft Xbox One controller as a simple Sensory Substitution and Augmentation device, and detailed the process utilised for its preparation as an intuitive and versatile interface for such tasks.

To reiterate the words of Hanneton, Auvray and Durette [17], echoed by Lobo [32], we run the risk of short term studies in HCI prioritising the discovery of significant comparisons over true advancements in the field, that is, creation of devices and interfaces which genuinely enhance user experience. We hope that the presentation of a simple yet effective feedback condition, using an off-the-shelf device in a medium-term study enables and promotes the creation and demonstration of such interfaces in the future. 


\section{REFERENCES}

[1] Malika Auvray, Sylvain Hanneton, and J. Kevin O’Regan. 2007. Learning to perceive with a visuo-auditory substitution system: Localisation and object recognition with 'The vOICe'. Perception 36, 3 (2007), 416-430. https://doi.org/10.1068/p5631

[2] Paul Bach-y Rita. 1972. Brain mechanisms in sensory substitution. Academic Press, New York. 182 pages.

[3] Matthias Berning, Florian Braun, Till Riedel, and Michael Beigl. 2015. ProximityHat: A Head-Worn System for Subtle Sensory Augmentation with Tactile Stimulation. In Proceedings of the 2015 ACM International Symposium on Wearable Computers (Osaka, Japan) (ISWC '15). Association for Computing Machinery, New York, NY, USA, 31-38. https://doi.org/10.1145/2802083.2802088

[4] Craig Bertram and Tom Stafford. 2016. Improving training for sensory augmentation using the science of expertise. Neuroscience and Biobehavioral Reviews 68 (2016), 234-244. https://doi.org/10.1016/j.neubiorev.2016.05.026

[5] Stephen Brewster and Lorna M. Brown. 2004. Tactons: Structured Tactile Messages for Non-Visual Information Display. In Proceedings of the Fifth Conference on Australasian User Interface - Volume 28 (Dunedin, New Zealand) (AUIC '04). Australian Computer Society, Inc., AUS, 15-23.

[6] Anthony Carton and Lucy E. Dunne. 2013. Tactile Distance Feedback for Firefighters: Design and Preliminary Evaluation of a Sensory Augmentation Glove. In Proceedings of the 4th Augmented Human International Conference (Stuttgart, Germany) (AH '13). Association for Computing Machinery, New York, NY, USA, 58-64. https://doi.org/10.1145/2459236.2459247

[7] Alvaro Cassinelli, Carson Reynolds, and Masatoshi Ishikawa. 2006. Haptic radar/extended skin project. ACM SIGGRAPH 2006: Sketches, SIGGRAPH '06 1 (2006), 1991. https://doi.org/10.1145/1179849.1179892

[8] Jennifer Chen, Pablo Castillo, Robert Turcotte, Ali Israr, and Frances Lau. 2018. Feeling Speech on the Arm. https://doi.org/10.1145/3170427.3186537

[9] James C. Craig. 1972. Difference threshold for intensity of tactile stimuli. Perception \& Psychophysics 11, 2 (1972), 150-152. https://doi.org/10.3758/ BF03210362

[10] Ophelia Deroy and Malika Auvray. 2014. Perception and its modalities. Oxford University Press Oxford, UK, 198 Madison Avenue, New York, NY 10016, Chapter A crossmodal perspective on sensory substitution, 327-349.

[11] Gershon Dublon and Joseph A. Paradiso. 2012. Tongueduino: Hackable, High-Bandwidth Sensory Augmentation. In CHI '12 Extended Abstracts on Human Factors in Computing Systems (Austin, Texas, USA) (CHI EA '12). Association for Computing Machinery, New York, NY, USA, 1453-1454. https://doi.org/10.1145/2212776.2212482

[12] Gustav Theodor Fechner. 1860. Elemente der psychophysik. Vol. 2. Breitkopf u. Härtel, Leipzig.

[13] Nazim Gizem Forta, Miyuki Morioka, and Michael J Griffin. 2009. Difference thresholds for the perception of whole-body vertical vibration: Dependence on the frequency and magnitude of vibration. Ergonomics 52, 10 (2009), 1305-1310. https://doi.org/10.1080/00140130903023709

[14] Tom Froese, Marek McGann, William Bigge, Adam Spiers, and Anil K. Seth. 2012. The enactive torch: A new tool for the science of perception. IEEE Transactions on Haptics 5, 4 (2012), 365-375. https://doi.org/10.1109/TOH.2011.57

[15] Monica Gori, Giulia Cappagli, Alessia Tonelli, Gabriel Baud-Bovy, and Sara Finocchietti. 2016. Devices for visually impaired people: High technological devices with low user acceptance and no adaptability for children. Neuroscience and Biobehavioral Reviews 69 (2016), $79-88$. https://doi.org/10.1016/j.neubiorev.2016.06.043

[16] Giles Hamilton-Fletcher, Thomas D. Wright, and Jamie Ward. 2016. Cross-modal correspondences enhance performance on a colour-to-sound sensory substitution device. Multisensory Research 29, 4-5 (2016), 337-363. https://doi.org/10.1163/22134808-00002519

[17] Sylvain Hanneton, Malika Auvray, and Barthélemy Durette. 2010. The Vibe: A versatile vision-to-audition sensory substitution device. Applied Bionics and Biomechanics 7, 4 (2010), 269-276. https://doi.org/10.1080/11762322.2010.512734

[18] Sandra G. Hart. 2006. Nasa-Task Load Index (NASA-TLX); 20 Years Later. Proceedings of the Human Factors and Ergonomics Society Annual Meeting 50, 9 (2006), 904-908. https://doi.org/10.1177/154193120605000909

[19] HTC. 2020. VIVE Cosmos Overview. HTC. https://www.vive.com/us/product/vive-cosmos/overview/

[20] Michaël Huet, Cyril Camachon, Laure Fernandez, David M. Jacobs, and Gilles Montagne. 2009. Self-controlled concurrent feedback and the education of attention towards perceptual invariants. Human Movement Science 28, 4 (2009), 450-467. https://doi.org/10.1016/j.humov.2008.12.004

[21] Inwook Hwang, Jongman Seo, Myongchan Kim, and Seungmoon Choi. 2013. Vibrotactile perceived intensity for mobile devices as a function of direction, amplitude, and frequency. IEEE Transactions on Haptics 6, 3 (2013), 352-362. https://doi.org/10.1109/TOH.2013.2

[22] Lynette A. Jones. 2011. Tactile communication systems. optimizing the display of information. Progress in Brain Research 192 (2011), $113-128$. https://doi.org/10.1016/B978-0-444-53355-5.00008-7

[23] Árni Kristjánsson, Alin Moldoveanu, Ómar I. Jóhannesson, Oana Balan, Simone Spagnol, Vigdís Vala Valgeirsdóttir, and Rúnar Unnthorsson. 2016. Designing sensory-substitution devices: Principles, pitfalls and potential1. Restorative Neurology and Neuroscience 34, 5 (2016), $769-787$. https://doi.org/10.3233/RNN-160647

[24] Matti Krüger, Tom Driessen, Christiane B Wiebel-Herboth, Joost CF de Winter, and Heiko Wersing. 2020. Feeling Uncertain-Effects of a Vibrotactile Belt that Communicates Vehicle Sensor Uncertainty. Information 11, 7 (2020), 353.

[25] Matti Krüger, Christiane B. Wiebel-Herboth, and Heiko Wersing. 2020. The Lateral Line: Augmenting Spatiotemporal Perception with a Tactile Interface. In Proceedings of the Augmented Humans International Conference (Kaiserslautern, Germany) (AHs '20). Association for Computing Machinery, New York, NY, USA, Article 6, 10 pages. https://doi.org/10.1145/3384657.3384775

[26] Ben Kuchera. 2017. How the Nintendo Switch's HD rumble makes Tumbleseed feel real. Polygon. https://www.polygon.com/2017/5/1/15499328/ tumbleseed-hd-rumble-nintendo-switch

Manuscript submitted to ACM 
[27] Sabine U. König, Frank Schumann, Johannes Keyser, Caspar Goeke, Carina Krause, Susan Wache, Aleksey Lytochkin, Manuel Ebert, Vincent Brunsch, Basil Wahn, Kai Kaspar, Saskia K. Nagel, Tobias Meilinger, Heinrich Bülthoff, Thomas Wolbers, Christian Büchel, and Peter König. 2016. Learning New Sensorimotor Contingencies: Effects of Long-Term Use of Sensory Augmentation on the Brain and Conscious Perception. PLOS ONE 11, 12 (12 2016), 1-35. https://doi.org/10.1371/journal.pone.0166647

[28] Charles Lenay, Olivier Gapenne, Sylvain Hanneton, Catherine Marque, and Christelle Genouëlle. 2003. Sensory Substitution : Limits and Perspectives. Touching for Knowing Cognitive psychology of haptic manual perception 19 (2003), 275-292. http://books.google.com/books?hl=fr $\{\&\} \mathrm{lr}=\{\&\} \mathrm{id}=$ GSOhMpdyobAC $\{\&\}$ pgis $=1$

[29] Shelly Levy-Tzedek, Itai Novick, Roni Arbel, Sami Abboud, Shachar Maidenbaum, Eilon Vaadia, and Amir Amedi. 2012. Cross-sensory transfer of sensory-motor information: visuomotor learning affects performance on an audiomotor task, using sensory-substitution. Scientific reports 2 (2012), 949.

[30] Lorena Lobo. 2019. Current alternatives on perceptual learning: introduction to special issue on post-cognitivist approaches to perceptual learning. Adaptive Behavior 27, 6 (2019), 355-362. https://doi.org/10.1177/1059712319875147

[31] Lorena Lobo, Patric C. Nordbeck, Vicente Raja, Anthony Chemero, Michael A. Riley, David M. Jacobs, and D. Travieso. 2019. Route selection and obstacle avoidance with a short-range haptic sensory substitution device. International fournal of Human Computer Studies 132, March (2019), 25-33. https://doi.org/10.1016/j.ijhcs.2019.03.004

[32] Lorena Lobo, David Travieso, Antonio Barrientos, and David M Jacobs. 2014. Stepping on obstacles with a sensory substitution device on the lower leg: Practice without vision is more beneficial than practice with vision. Plos one 9, 6 (2014), e98801.

[33] Shachar Maidenbaum, Sami Abboud, and Amir Amedi. 2014. Sensory substitution: Closing the gap between basic research and widespread practical visual rehabilitation. Neuroscience and Biobehavioral Reviews 41 (2014), 3-15. https://doi.org/10.1016/j.neubiorev.2013.11.007 arXiv:arXiv:1011.1669v3

[34] Shachar Maidenbaum, Shelly Levy-Tzedek, Daniel Robert Chebat, and Amir Amedi. 2013. Increasing accessibility to the blind of virtual environments, using a virtual mobility aid based on the "EyeCane": Feasibility study. PLoS ONE 8, 8 (2013), 1-7. https://doi.org/10.1371/journal.pone.0072555

[35] Peter B L Meijer. 1992. An Experimental System for Auditory Image Representations. IEEE Transactions on Biomedical Engineering 39, 2 (1992), 112-121. https://doi.org/10.1109/10.121642

[36] Alessandro Moscatelli, Maura Mezzetti, and Francesco Lacquaniti. 2012. Modeling psychophysical data at the population-level: The generalized linear mixed model. Journal of Vision 12, 11 (2012), 1-17. https://doi.org/10.1167/12.11.26

[37] David Myers. 2004. Tactile Situational Awareness System ( TSAS ). , 5 pages. https://www.sbir.gov/sbirsearch/detail/124876

[38] Saskia K. Nagel, Christine Carl, Tobias Kringe, Robert Märtin, and Peter König. 2005. Beyond sensory substitution - Learning the sixth sense. Fournal of Neural Engineering 2, 4 (2005), 13-26. https://doi.org/10.1088/1741-2560/2/4/R02

[39] Neosensory. 2020. Neosensory - Experience sound as touch and gain new awareness. Neosensory. https://neosensory.com/

[40] Scott D. Novich and David M. Eagleman. 2015. Using space and time to encode vibrotactile information: toward an estimate of the skin's achievable throughput. Experimental Brain Research 233, 10 (2015), 2777-2788. https://doi.org/10.1007/s00221-015-4346-1

[41] Oculus. 2020. Oculus Rift: VR Headset for VR Ready PCs. Oculus. https://www.oculus.com/rift/

[42] J Kevin O’Regan and Alva Noë. 2001. A sensorimotor account of vision and visual consciousness. Behavioral and brain sciences 24, 5 (2001), 939.

[43] Michael J. Proulx, David J. Brown, Achille Pasqualotto, and Peter Meijer. 2014. Multisensory perceptual learning and sensory substitution. Neuroscience and Biobehavioral Reviews 41 (2014), 16-25. https://doi.org/10.1016/j.neubiorev.2012.11.017

[44] Michael J Proulx, Petra Stoerig, Eva Ludowig, and Inna Knoll. 2008. Seeing 'where'through the ears: effects of learning-by-doing and long-term sensory deprivation on localization based on image-to-sound substitution. PloS one 3, 3 (2008), e1840.

[45] Matteo Rossi, Matteo Bianchi, Edoardo Battaglia, Manuel G. Catalano, and Antonio Bicchi. 2019. HapPro: A Wearable Haptic Device for Proprioceptive Feedback. IEEE Transactions on Biomedical Engineering 66, 1 (2019), 138-149. https://doi.org/10.1109/TBME.2018.2836672

[46] Carlos Sainz Martinez and Faustina Hwang. 2015. TESSA -Toolkit for experimentation with multimodal sensory substitution and augmentation. Proceedings of the 33rd Annual ACM Conference Extended Abstracts on Human Factors in Computing Systems - CHI EA '15 2 (2015), $259-262$. https://doi.org/10.1145/2702613.2725451

[47] B. F. Van Erp Jan, A. H.C. Van Veen Hendrik, and Ruijsendaal Mark. 2007. More than a feeling: Bringing touch into astronauts' spatial orientation. Microgravity Science and Technology 19, 5-6 (2007), 108-112. https://doi.org/10.1007/BF02919463

[48] Jamie Ward and Peter Meijer. 2010. Visual experiences in the blind induced by an auditory sensory substitution device. Consciousness and Cognition 19, 1 (2010), 492-500. https://doi.org/10.1016/j.concog.2009.10.006

[49] Jamie Ward and Thomas Wright. 2014. Sensory substitution as an artificially acquired synaesthesia. Neuroscience and Biobehavioral Reviews 41 (2014), 26-35. https://doi.org/10.1016/j.neubiorev.2012.07.007

[50] Doruk Yildirim, Luis Edgardo Fraguada, and Elizabeth Esther Bigger. 2019. DualSkin: Ambient Electric Field Sensing Wearable. In Proceedings of the 23rd International Symposium on Wearable Computers (London, United Kingdom) (ISWC '19). Association for Computing Machinery, New York, NY, USA, 339-345. https://doi.org/10.1145/3341163.3346931

[51] Siyan Zhao, Ali Israr, Frances Lau, and Freddy Abnousi. 2018. Coding Tactile Symbols for Phonemic Communication. In Proceedings of the 2018 CHI Conference on Human Factors in Computing Systems (Montreal QC, Canada) (CHI '18). Association for Computing Machinery, New York, NY, USA, 1-13. https://doi.org/10.1145/3173574.3173966 


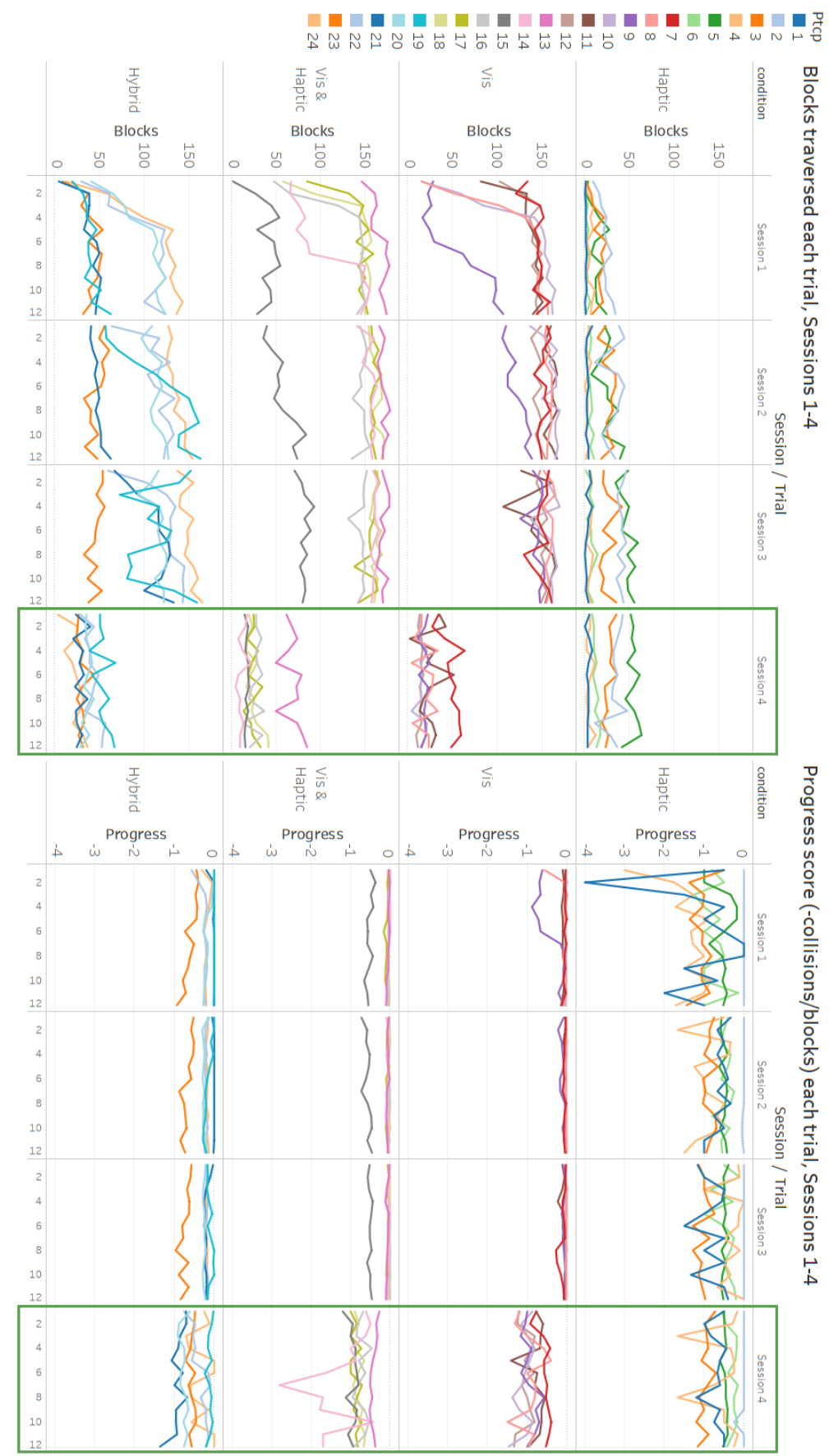

Fig. 20. Chart showing each participant's number of blocks traversed (left) and progress score (right) during each trial, across all four sessions. Data from sessions 1-3 not used in further analysis as conditions render metrics incomparable. In session 4 (highlighted green), all participants performed the task under identical conditions (haptic feedback only), allowing comparative analysis.

Manuscript submitted to ACM 